Draft VERsion April 17, 2018

Preprint typeset using $\mathrm{LATE}_{\mathrm{E}} \mathrm{X}$ style emulateapj v. 12/16/11

\title{
ULTRA-SHORT-PERIOD PLANETS FROM SECULAR CHAOS
}

\author{
Cristobal Petrovich ${ }^{1,2}$, Emily Deibert ${ }^{3}, \&$ Yanqin $\mathrm{Wu}^{3}$ \\ Draft version April 17, 2018
}

\begin{abstract}
Over a hundred rocky planets orbiting Sun-like stars in very short orbital periods $(\lesssim 1$ day) have been discovered by the Kepler mission. These planets, known as ultra-short-period (USP) planets, are unlikely to have formed locally, or have attained their current orbits when their birth protoplanetary disks were still present. Instead, they must have migrated in later in life. Here we propose that these planets reach their current orbits by high-eccentricity migration. In a scaled-down version of the dynamics that may have been experienced by their high mass analog, the hot Jupiters, these planets reach high eccentricities via chaotic secular interactions with their companion planets and then undergo orbital circularization due to dissipation of tides raised on the planet. This proposal is motivated by the following observations: planetary systems observed by Kepler often contain several super-Earths with non-negligible eccentricities and inclinations, and possibly extending beyond $\sim$ AU distances; while only a small fraction of USP planets have known transiting companions, and none closely spaced, we argue that most of them should have companions at periods of $\sim 10-50$ days. The outer sibling planets, through secular chaos, can remove angular momentum from the inner most planet, originally at periods of $\sim 5-10$ days. When the latter reaches an eccentricity higher than 0.8 , it is tidally captured by the central star and becomes an USP planet. This scenario naturally explains the observation that most USP planets have significantly more distant transiting companions compared to their counterparts at slightly longer periods ( $1-3$ days), a feature un-accounted for in other proposed scenarios. Our model also predicts that USP planets should have: (i) spin-orbit angles, and inclinations relative to outer planets, in the range of $\sim 10^{\circ}-50^{\circ}$; (ii) several outer planetary companions extending to beyond $\sim 1$ AU distances, both of which may be tested by TESS and its follow-up observations.

Subject headings: planets and satellites: dynamical evolution and stability
\end{abstract}

\section{INTRODUCTION}

Ultra-short-period planets (or USP planets), the rare and enigmatic class of transiting exoplanets with orbital periods shorter than one day, have an unknown origin and are the topic of this study. For reference, a 1 day orbital period for a solar-type star corresponds to $a \approx$ $0.02 \mathrm{AU}$ and a blackbody temperature of $T \approx 2100 \mathrm{~K}$.

About a hundred of these planets have been discovered by the Kepler transit mission, and they are inferred to exist around $\sim 0.5 \%$ of stars (Sanchis-Ojeda et al. 2014), making them slightly less abundant than hot Jupiters (Jovian planets orbiting closer than 10 days, frequency $\sim 1 \%$, e.g., Mayor et al. 2011, Howard et al. 2012, Wright et al. 2012). These planets appear statistically different from the more populous Kepler systems, their closest analog, in that they are either the only transiting planet in the system, or in cases when they have transiting outer companions (e.g., in Kepler-10 and $\rho 55$ Cancri systems, Batalha et al. 2011, Butler et al. 1997; Marcy et al. 2002; McArthur et al. 2004; Fischer et al.|2008), the latter orbit at periods $\gtrsim 10$ times longer (Steffen \& Coughlin 2016), i.e., much further away than in typical

\footnotetext{
${ }^{1}$ Canadian Institute for Theoretical Astrophysics, University of Toronto, 60 St. George Street, Toronto, Ontario, M5S 1A7, Canada

${ }^{2}$ Centre for Planetary Sciences, Department of Physical \& Environmental Sciences, University of Toronto at Scarborough, Toronto, Ontario M1C 1A4, Canada

3 Department of Astronomy \& Astrophysics, University of Toronto, 50 St. George Street, Toronto, Ontario, M5S 3H4, Canada
}

Kepler multi-planet systems.

It is almost certain that USP planets did not form at their current locations. These planets fall within the dust sublimation radius for even the most refractive minerals (iron sublimates at $T \approx 2000 \mathrm{~K}$ ). Moreover, the radii of host stars during the pre-main sequence phase were several times larger than their current values (e.g., Palla \& Stahler 1991; D'Antona \& Mazzitelli 1994) and would have swallowed a number of the closer-in USP planets.

Several formation models have been proposed to explain the origin of these planets, with varying degrees of success. One theory is that they are the exposed cores of giant planets after their gaseous atmospheres have been stripped off by photo-evoporation or tidal forces (Jackson et al. 2013, Valsecchi et al. 2014, Jackson et al. 2016). While there are theoretical objections to this scenario (e.g., Murray-Clay et al. 2009), empirically, Winn et al. (2017) compared the metallicities of stars harboring USP planets and hot Jupiters and found that they are significantly different, with the hot Jupiters preferentially orbiting around metal-rich stars Gonzalez 1997 Santos et al. 2004, Fischer \& Valenti 2005). Instead, the USP planets' hosts' metallicities are indistinguishable from those of Kepler planets' hosts, for which there is no notable association with high metallicity (Udry et al. 2006, Schlaufman \& Laughlin 2011, Buchhave et al. 2012). They went on to posit that USP planets may be the exposed cores of Neptunes (Valencia et al. 2010, Owen \& Wu 2013, Lundksvist et al. 2016; Lee \& Chiang 2017). However, even if the latter proposal is correct, one 
still needs to understand how the planets get so close to the stars in the first place. Proposals like that in Mandell et al. (2007), where USP planets are formed from the accretion of material "shepherded" inwards by outer giant planets, suffer similar setbacks.

In this work, we propose that most of the ultra-shortperiod planets were initially the innermost planets in typical multi-planet Kepler systems. They reach their current orbits from a combination of secular chaotic excitation of their eccentricities and efficient tidal dissipation in the planets at high eccentricities. This is akin to one of the proposals to form hot Jupiters, secular chaos ( $\mathrm{Wu} \&$ Lithwick 2011). Consider a planetary system with a large number of planets $(N \geq 3)$. If these planets are spaced far enough from each other such that their interactions are mostly secular in nature (as opposed to mean-motion resonances), and if the orbits of these planets have some moderate amounts of eccentricity and inclination, secular interactions can become non-periodic and chaotic, leading to diffusive angular momentum transfer among planets that tends to raise the eccentricity and inclination of the innermost planet (Laskar 1996; Lithwick \& $\mathrm{Wu} 2011,2014)$. As this planet's pericenter approaches the central star with an ever-decreasing range, tidal interactions enter at some point. This dissipates the orbital energy of the planet, bringing it to close circular orbits around the star, and snatching it away from the forcing by other planets. Eventually, we are left with a planet that is orbiting at a close range from the star and is dynamically detached from the outer system.

This proposal is motivated by multiple lines of arguments:

1. analogy with hot Jupiters - there are many observational parallels between USP planets and hot Jupiters. Both are rare classes compared to their more populous cousins: hot Jupiters occur in $\sim 1 \%$ of FGK stars, while cold Jupiters occur around $10-15 \%$; USP planets occur in $\sim 0.5-1 \%$ of stars, while Kepler systems (planets with radii less than that of Neptune and inward of 400 days) occur around 30\% of stars (Zhu et al. 2018). They also tend to have lower masses compared to these cousins: USP planets have radii $R_{p} \approx 0.8-1.2 R_{\oplus}$, on the low end of the size spectrum even among those closein Kepler planets that have presumably suffered photoevaporation and are bare rocky cores; while masses of hot Jupiters are a factor of $\sim 2$ or more below the average cold Jupiters (e.g., Dawson \& Johnson 2018). Lastly, they both lack close neighbors: hot Jupiters have been known to be mostly 'lonely' (e.g., Steffen et al. 2012, Huang et al. 2016), though lately they have been shown to possess an abundance of distant 'friends' (Knutson et al. 2014); analogously, most USP planets are either apparently single or have distant (orbits of tens of days) outer companions (Steffen \& Coughlin 2016). These similarities propel us to invoke a common mechanism for their formation.

2. high-multiplicity systems are common - about $30 \%$ of stars host low-mass, multi-planet systems inward of 400 days, with an average multiplicity of 3 (Zhu et al. 2018). Further than 400 days, transit searches are highly incomplete, but there is evidence suggesting that the planet ladder goes on (Foreman-Mackey et al.2016). Microlens-

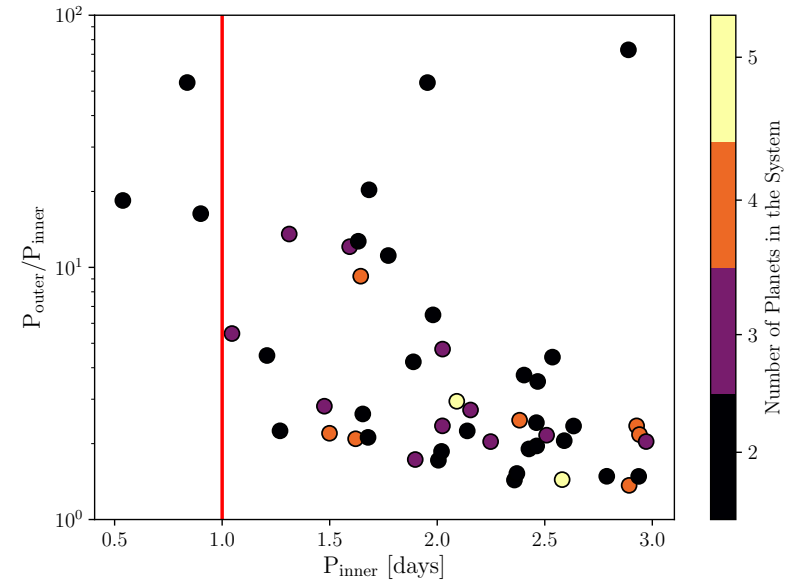

FIG. 1.- Period ratios of adjacent transiting planets where the inner planet has a period less than 3 days and the stellar masses are greater than $0.75 M_{\odot}$. The color coding indicates the number of planets transiting in each system. The 3 (out of 44) ultra-shortperiod planets (left of vertical line) with outer companions have period ratios $\gtrsim 15$, while the planets in slightly longer periods have a broader range, including more compact configurations.

ing observations also show that Neptunes are common in long-period orbits, at least around M-dwarfs (e.g., Suzuki et al. 2016).

3. multi-planet systems often have significant eccentricities and inclinations - the Kepler sample shows that systems hosting three or fewer planets in sub-year orbits (also those most likely to interact secularly) have significant inclination dispersions $\left(i_{\mathrm{rms}} \equiv<i^{2}>^{1 / 2} \sim 0.1\left(5.7^{\circ}\right)\right.$; Zhu et al. 2018). These systems are generally observed as single-transiting systems. Interestingly, these very systems also appear to exhibit large eccentricity dispersions $\left(e_{\mathrm{rms}} \equiv<e^{2}>^{1 / 2} \gtrsim 0.1\right.$; Xie et al. 2016).

This paper is organized as follows. In $\$ 2$ we present the sample of USP planets discovered by the Kepler survey and study the possible orbital properties of their outer planetary companions. In $\$ 3$ we set analytical constraints on our proposed mechanism and in 84 we present the results of some numerical experiments. Finally, \$5 provides a discussion of our results and a critique of previous works, and we summarize our main findings in $\$ 6$.

\section{ULTRA-SHORT-PERIOD PLANETS LIKELY HAVE DISTANT COMPANIONS}

Among the currently known USP planets, only a small fraction are in multiple-transiting systems. We use this to infer how likely USP planets are to have distant companions, and the orbital configurations (in terms of orbital periods and mutual inclinations) of these companions. We conclude that most should have companions, but the outer companions are either distant $(P \gtrsim 20$ days), or are highly mutually inclined $\left(i_{\mathrm{rms}} \gtrsim 10^{\circ}\right)$.

\subsection{The sample of very-short-period planets $(P<3$ days)}

In Table 1 we show the number of stars with single- and multi-transiting planets discovered by Kepler with $R_{p}<$ $4 R_{\oplus}$ and $P \leq 3$ days from the NASA exoplanet archive 

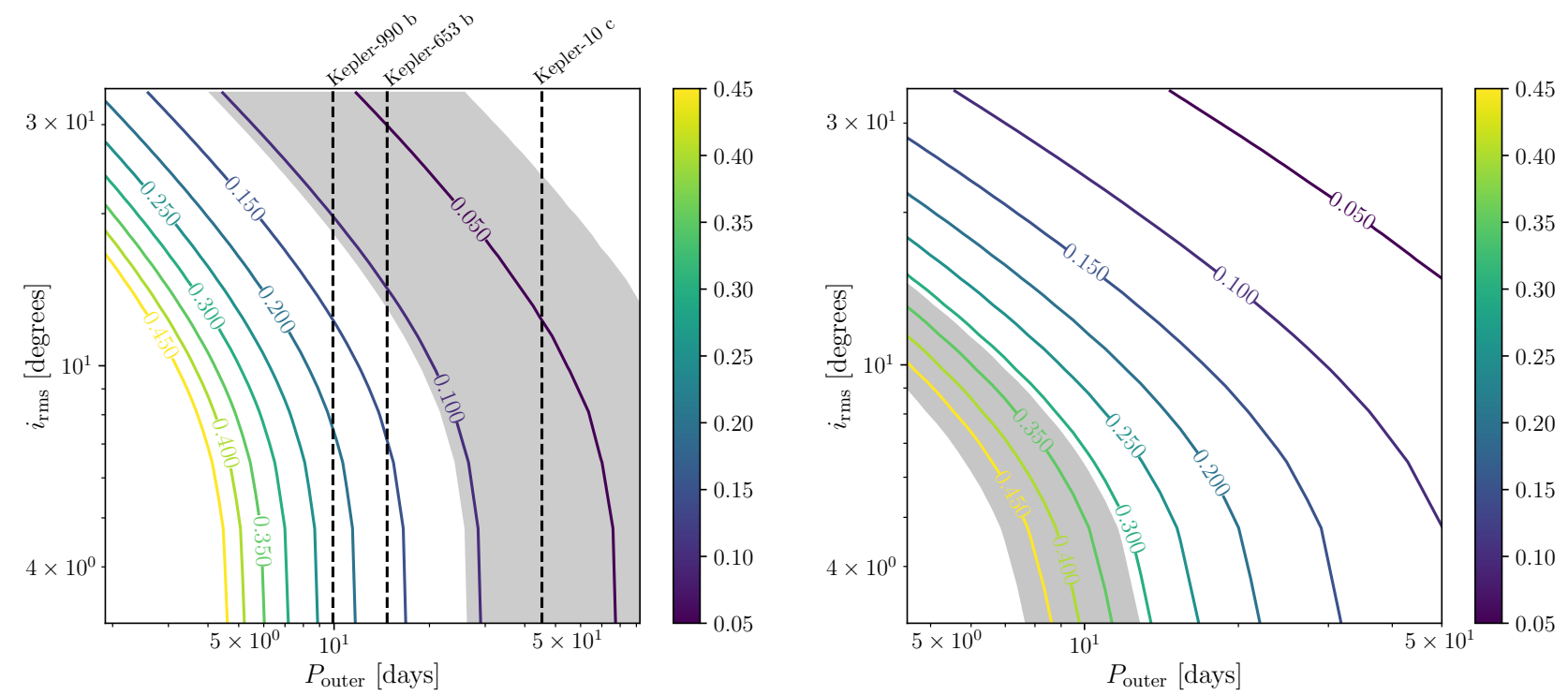

FIG. 2.- Expected multiplicity ratio $\left(\left\langle f_{>1} / f_{1}\right\rangle\right.$ in Equation 2] for different inclination dispersions drawn from a Fischer distribution and periods of outer nearest neighbor. Left panel: the inner planet is an USP planet ( $P_{\text {inner }}<1$ day). Right panel: we set $P_{\text {inner }} \in[1,2]$ days. The inner planets are assumed to have one extra outer companion in the indicated period range. The shaded region indicates the observed ratio with Poisson error, showing that the outer companions of USP planets are likely beyond $\sim 20$ days.

TABLE 1

MULTIPLICITY RATIO FOR VERY-SHORT-PERIOD PLANETS WITH $R_{p}<4 R_{\oplus}$ DISCOVERED BY Kepler

\begin{tabular}{c|c|c}
\hline \hline inner period & $f_{>1} / f_{1}=\#$ mult./\#sing. & $f_{>1} / f_{1}\left(M_{s} \geq 0.75 M_{\odot}\right)$ \\
\hline$<1$ days & $7 / 55 \simeq 0.13 \pm 0.051$ & $3 / 44 \simeq 0.068 \pm 0.041$ \\
$1-2$ days & $28 / 53 \simeq 0.53 \pm 0.12$ & $18 / 44 \simeq 0.41 \pm 0.11$ \\
$2-3$ days & $50 / 66 \simeq 0.76 \pm 0.14$ & $31 / 54 \simeq 0.58 \pm 0.13$ \\
\hline
\end{tabular}

as of January 2018 . We notice that the number of stars with single-transiting planets remains roughly constant for the different bins, while the number of stars with multi-transiting planets increases abruptly with period.

By looking in more detail at the sample of 7 systems with USP planets in multi-transiting systems, we notice that there seem to be two distinct classes depending on the stellar types:

- for $M_{s} \lesssim 0.75 M_{\odot}$ there are 4 systems (out of 11 ) in preferentially compact configurations, including Kepler-42 (3 planets with $\left.P_{\text {out }} / P_{\mathrm{USP}} \simeq 2.7\right)$, Kepler-32 (5 planets with $\left.P_{\text {out }} / P_{\text {USP }} \simeq 3.9\right)$, and Kepler-80 $\left(6\right.$ planets with $\left.P_{\text {out }} / P_{\text {USP }} \simeq 3.1\right)$. The exception to this trend is Kepler-732 (2 planets with $\left.P_{\text {out }} / P_{\mathrm{USP}} \simeq 10\right)$.

- for $M_{s} \gtrsim 0.75 M_{\odot}$ there are 3 systems (out of 44 ) with dynamically detached USP planets, including Kepler-10 (2 planets with $\left.P_{\text {out }} / P_{\text {USP }} \simeq 54\right)$, Kepler-653 (2 planets with $\left.P_{\text {out }} / P_{\mathrm{USP}} \simeq 16\right)$, and Kepler-990 (2 planets with $\left.P_{\text {out }} / P_{\mathrm{USP}} \simeq 18\right)$.

The first class is reminiscent of M-dwarfs which are known to have more miniature systems (Dressing \& Charbonneau 2015). Although the USP planets are still more detached compared to other planets, we ignore this class here and focus instead on systems around FGK stars. Thus, we apply an arbitrary cut in host stellar

\footnotetext{
4 Confirmed planets from NASA exoplanet archive based on Quarters 1-17, https://exoplanetarchive.ipac.caltech.edu
}

mass of $M_{s} \geq 0.75 M_{\odot}$, which is roughly equivalent to making a cut in effective temperature of $T_{\text {eff }} \gtrsim 4600 \mathrm{~K}$. This sample contains 3 USP planets in multi-transiting systems and 44 in single-transiting systems.

Defining a multiplicity ratio

$$
\frac{f_{>1}}{f_{1}} \equiv \frac{\# \text { multi transiting }}{\# \text { single transiting }}
$$

we have $f_{>1}^{\text {USP }} / f_{1}^{\text {USP }}=3 / 44 \simeq 0.068 \pm 0.041$ for our USP planets. In contrast, systems with an inner planet at $P \in[1,3]$ days have $f_{>1} / f_{1}=49 / 98=0.5 \pm 0.087$.

We note that the recent study by Adams et al. (2016) including various transit surveys (Kepler, K2, WASP) finds a similar ratio of $f_{>1}^{\mathrm{USP}} / f_{1}^{\mathrm{USP}}=11 / 164 \simeq 0.067 \pm$ 0.021 . Similarly, the previous work by Sanchis-Ojeda et al. (2014) analyzing the Kepler data with their own detection pipeline finds a slightly larger number of companions with $f_{>1}^{\mathrm{USP}} / f_{1}^{\mathrm{USP}}=10 / 59 \simeq 0.17 \pm 0.06$, consistent with our sample without the host star mass cut $(7 / 55 \simeq 0.13 \pm 0.051)$ and marginally consistent with the systems with $M_{s} \geq 0.75 M_{\odot}$.

The multiplicity ratio for USP planets $(0.068$ for our preferred sample) is so low one may worry that many USP planets are truly singles. However, this is unlikely (also see Steffen \& Hwang 2015, Steffen \& Coughlin 2016). Even if every USP planet has a companion, but at large periods as is observed for the 3 systems, the chance of observing the companion transit is low. This is made worse if the mutual inclinations are large. This is quantified in Figure 2 and discussed in detail below. Moreover, since the USP planets are rare with an occurrence rate nearly two orders of magnitude lower than that of average Kepler systems (Sanchis-Ojeda et al.2014), which are in turn very common and harbor multiple planets (Zhu et al. 2018), it seems reasonable to investigate whether some unusual architecture in the latter systems can lead to USP planet formation, as opposed to assuming that USP planets are a class of their own and are truly single. 


\subsection{Constraints from period ratios}

In Figure 11 we show the period ratios of adjacent planets for systems with an inner planet inside a 2 day orbit. The set of three USP planets have periods ratios $P_{\text {outer }} / P_{\text {inner }} \gtrsim 15$, while the planets in the period range of $1-2$ days have a median period ratio of $P_{\text {outer }} / P_{\text {inner }} \sim 5$. This result indicates that the USP planets are more dynamically detached than their widerorbit counterparts.

This result that very-short-period planets have larger periods ratios was previously pointed out by Steffen \& Farr (2013) and the authors were able to place a boundary at $P_{\text {outer }} / P_{\text {inner }} \gtrsim 2.3\left(P_{\text {inner }} / \text { day }\right)^{-2 / 3}$. Their results are based on the Quarters 1-12 KOI catalog, while ours are based on the Quarters 1-17 catalog with confirmed planets and a cut in host star mass (see previous section).

\subsection{Constraints from multiplicity ratios: $f_{>1} / f_{1}$}

We compute the multiplicity ratio $f_{>1}^{\mathrm{USP}} / f_{1}^{\mathrm{USP}}$ expected by Kepler assuming that all systems with USP planets have at least one companion inside $\sim 50$ days.

We shall assume that for host stars with detected USP planets, the Kepler pipeline has a high detection efficiency at detecting planets with $R \gtrsim 1 R_{\oplus}$ in the period range of $P \sim 1-50$ days and the ratio $f_{1}^{\mathrm{USP}} / f_{>1}^{\mathrm{USP}}$ mainly depends on the transit probabilities. This is a reasonable assumption as the average detection probability of planets with radii $R_{p} \gtrsim 2 R_{\oplus}\left(R_{p} \gtrsim 1 R_{\oplus}\right)$ with periods $\lesssim 50$ days is near unity ( $\gtrsim 50 \%$ ) (e.g., Burke et al. 2015; Petigura et al. 2017).

We estimate the transit probabilities following the method and notation in Tremaine \& Dong (2011) and provide the necessary details to reproduce our results in the Appendix. From Equation A9 we compute the expected multiplicity ratio assuming that the USP planet has one outer planetary companion as

$$
\left\langle\frac{f_{>1}}{f_{1}}\right\rangle=\frac{g_{22}\left(\epsilon_{\text {inner }}, \epsilon_{\text {outer }}, \kappa\right)}{\epsilon_{\text {inner }}-g_{22}\left(\epsilon_{\text {inner }}, \epsilon_{\text {outer }}, \kappa\right)},
$$

where $\epsilon_{\text {inner }}=R_{s} / a_{\text {inner }}, \epsilon_{\text {outer }}=R_{s} / a_{\text {outer }}$, and $\kappa$ is related to the mean-square value of $\sin i$ through Equation (A3). The function $g_{22}$ is given by Equation (A7).

In Figure 2 we show the contours of the multiplicity ratio from Equation (2) by integrating over the observed range of $\epsilon_{\text {inner }}$ for USP planets (left panel) and planets with in the period range of $1-2$ days (right panel). Thus, $\left\langle f_{>1} / f_{1}\right\rangle$ depends only on the orbital separation of the outer planets and the inclination dispersion $\left\langle\sin ^{2} i\right\rangle$ (or $\kappa$ through Eq. A3 $)$. As expected, $\left\langle f_{>1} / f_{1}\right\rangle$ decreases for higher inclination dispersions and longer-period outer planets. We include the $1-\sigma$ error bars for the observed ratio $f_{>1} / f_{1}$ from Table 1 with $M_{s} \geq 0.75 M_{\odot}$ and observe that the USP planets are constrained to large $i_{\text {rms }}$ and/or long $P_{\text {outer. }}$. In particular, if the $i_{\text {rms }} \lesssim 10^{\circ}$, then $P_{\text {outer }} \gtrsim 25$ days. If $P_{\text {outer }} \lesssim 10$ days, then $i_{\text {rms }} \gtrsim 20^{\circ}$.

Our estimate of $f_{>1}^{\mathrm{USP}} / f_{1}^{\mathrm{USP}}$ assumes that there are only two planets in the system. If the intrinsic multiplicity inside $\sim 50$ days is higher than 2 , then the expected ratio $f_{>1}^{\mathrm{USP}} / f_{1}^{\mathrm{USP}}$ would increase, demanding for longer periods and higher inclinations to match the observations. Therefore, considering only two planets is a conservative assumption to put constrains on the mini-

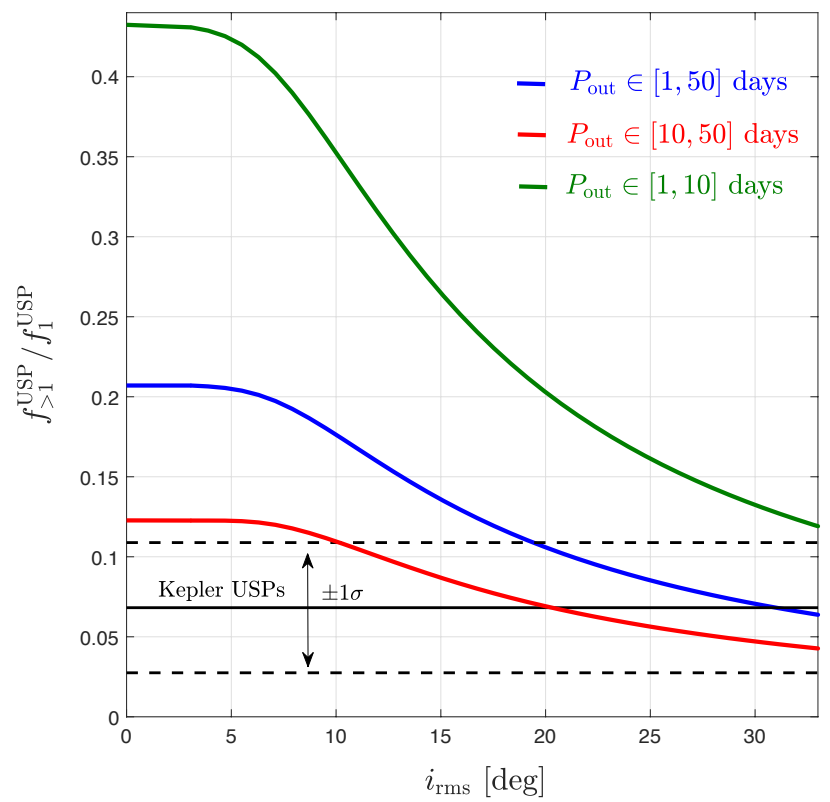

FIG. 3.- Expected multiplicity ratio $\left\langle f_{>1}^{\mathrm{USP}} / f_{1}^{\mathrm{USP}}\right\rangle$, for different inclination dispersions $\left(i_{\mathrm{rms}}\right)$ drawn from a Fischer distribution assuming that all USP planets have one companion in the period range indicated. The periods of the outer companion are drawn from the distribution in Equation 3 .

mum values of $P_{\text {outer }}$.

If we further assume that the outer companions do not know about the presence of an inner USP planet and they follow an orbital distribution from the Kepler sample (e.g., Tremaine \& Dong 2011)

$$
d p\left(\epsilon_{\text {outer }}\right) \propto \frac{\left(\epsilon_{\text {outer }} / 0.055\right)^{0.5}}{1+\left(\epsilon_{\text {outer }} / 0.055\right)^{3.6}} \frac{d \epsilon_{\text {outer }}}{\epsilon_{\text {outer }}},
$$

we can integrate $\left\langle f_{>1} / f_{1}\right\rangle$ in Equation (2) over $\epsilon_{\text {out }}$. We show the results for these multiplicity ratios in Figure 3 as a function of $i_{\text {rms }}$ and for different period ranges. We observe that if we limit the period range to $P_{\text {outer }}<10$ days (green line), then nearly isotropic inclination distributions are required to explain the data. For periodranges of $1-50$ days (solid blue line), a dispersion of $\sim 20^{\circ}$ or larger is required to explain the data while by limiting ourselves to $10-50$ days, then $\sim 20^{\circ}$ is preferred by the data.

In conclusion, the USP planets have outer companions with typical periods of $\sim 20-50$ days and/or $\sim 10$ days but which are very highly inclined $\left(i_{\text {rms }} \gtrsim 20^{\circ}\right)$. If the period of the outer planet is drawn from the observed distribution in Kepler up to 50 days, then the preferred inclination dispersion is $i_{\text {rms }} \gtrsim 20^{\circ}$.

\subsection{Comparison with very-short-period planets $(P \in[1,2]$ days $)$}

In the right panel of Figure 2 we show the contours of $\left\langle f_{>1} / f_{1}\right\rangle$ for planets $P \in[1,2]$ days. We observe that, unlike the USP planets (left panel), these systems are consistent with having at least one companion with $P_{\text {outer }} \lesssim 10$ (even down to $\sim 7$ days) in nearly coplanar orbits $\left(i_{\text {rms }} \lesssim 5^{\circ}\right)$.

We note, however, that the planet multiplicity inside 50 days might be higher than 2 planets (see Figure 11, 


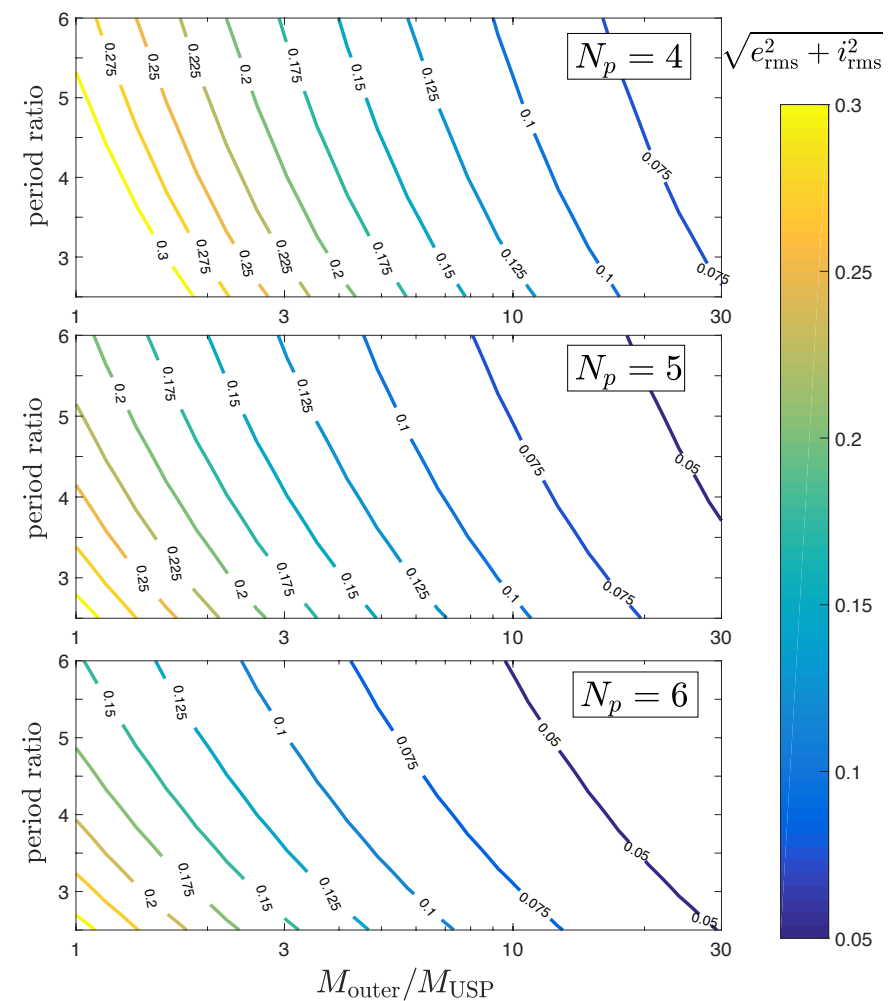

FIG. 4.- Contours of the minimum eccentricity and inclination dispersions expressed as $\left(e_{\mathrm{rms}}^{2}+i_{\mathrm{rms}}^{2}\right)^{1 / 2}$ to produce an USP planet by secular interactions (Eq. 77) as a function of the mass ratio between the USP planet and the outer planets, and the initial period ratios $P_{\mathrm{i}+1} / P_{i}\left(i=1 \ldots N_{\mathrm{p}}\right)$. Upper panel: total number of planets in the system $N_{\mathrm{p}}=4$. Middle panel: $N_{\mathrm{p}}=5$. Lower panel: $N_{\mathrm{p}}=6$.

in which case planets can be placed at larger orbital distances and still be consistent with the observed $f_{>1} / f_{1}$. This is unlikely as nearly half of the observed companions are inside 10 days (Figure 1).

\section{USP PLANETS PRODUCED BY SECULAR CHAOS: ANALYTICAL PRELIMINARIES}

Having argued that USP planets likely reside in multiplanet systems, we proceed to discuss other physical constraints in order for secular chaos to produce USP planets. We find that secular chaos can naturally lead to the formation of an USP planet in generic Kepler systems because:

- the requisite eccentricity and inclination values are likely common;

- precession from general relativity and tidal bulges can be overcome for planets initially orbiting beyond $\sim 5$-day obits;

- secular chaos excites the eccentricities slowly enough that tidal captures can occur, giving rise to planets with final periods of $\sim 1$ day.

In what follows, we justify each of these statements separately.

\subsection{Required angular momentum deficit to produce USP planets}

The angular momentum deficit (AMD), defined as follows (e.g., Laskar 1997)

$$
\begin{aligned}
\Lambda & =\left(G M_{s}\right)^{1 / 2} \sum_{i=1}^{N_{\mathrm{p}}} M_{i} \sqrt{a_{i}}\left[1-\sqrt{1-e_{i}^{2}} \cos i_{i}\right] \\
& \simeq\left(G M_{s}\right)^{1 / 2} \sum_{i=1}^{N_{\mathrm{p}}} \frac{1}{2} M_{i} \sqrt{a_{i}}\left[e_{i}^{2}+i_{i}^{2}\right],
\end{aligned}
$$

where $M_{s}$ is the stellar mass, and $M_{i}, a_{i}, e_{i}, i_{i}$ the mass, semi-major axis, eccentricity and inclination for planet $i$, describes the deficit in orbital angular momentum relative to that of a coplanar and circular system. AMD is an important index for the strength of secular interactions. Only when it exceeds a certain threshold can secular chaos occur. Moreover, there must be a minimum amount of AMD for the inner planet to be excited to a highly eccentric orbit and be tidally captured into a tight orbit. We consider this latter constraint below.

Since secular interactions do not modify the orbital energies, AMD is conserved. Thus, for the innermost planet to migrate from $a_{1}$ to a final circular orbit $a_{1, \mathrm{f}}$ (assuming angular momentum conservation during the circulation process, $\left.a_{1, \mathrm{f}}=a_{1}\left[1-e_{1}^{2}\right]\right)$, the minimum AMD required is

$$
\Lambda_{\min }=\left(G M_{s}\right)^{1 / 2} M_{i}\left[\sqrt{a_{1}}-\sqrt{a_{1, f}}\right],
$$

where we keep the term $\sqrt{a_{1, f}}$, ignored in the case of hot Jupiter migration (Wu \& Lithwick 2011) $\left(\sqrt{a_{1}}\right.$ is not generally much larger than $\sqrt{a_{1, f}}$ in our scenario). By setting $\Lambda_{\min }<\Lambda$ and assuming that all planetary orbits have some typical r.m.s. eccentricity $e_{\mathrm{rms}}=\left\langle e_{i}^{2}\right\rangle^{1 / 2}$ and inclination $i_{\mathrm{rms}}=\left\langle i_{i}^{2}\right\rangle^{1 / 2}$, we get the following condition for migration:

$$
e_{\mathrm{rms}}^{2}+i_{\mathrm{rms}}^{2} \leq \frac{2\left[1-\sqrt{a_{1, \mathrm{f}} / a_{1}}\right]}{\sum_{i=1}^{N_{\mathrm{p}}} \frac{M_{i}}{M_{1}} \sqrt{a_{\mathrm{i}} / a_{1}}} .
$$

Typically in our model, the planets start migration from $a_{1} \sim 0.05-0.1$ AU to $a_{1, \mathrm{f}} \sim 0.02 \mathrm{AU}$, so $\sqrt{a_{1} / a_{1, \mathrm{f}}} \sim$ 2 . By assuming that all the outer planets $(i>1)$ have the same mass $M_{\mathrm{i}}=M_{\text {outer }}$ and follow a simple spacing law with constant period ratio $\mathcal{P} \equiv P_{i+1} / P_{i}$, the above condition can be expressed as

$$
\begin{aligned}
\left(\frac{e_{\mathrm{rms}}}{0.1}\right)^{2}+ & \left(\frac{i_{\mathrm{rms}}}{0.1}\right)^{2} \simeq\left(\frac{M_{\mathrm{USP}}}{1 M_{\oplus}}\right)\left(\frac{10 M_{\oplus}}{M_{\text {outer }}}\right) \\
& \times\left[\frac{1}{10}\left(\frac{M_{\mathrm{USP}}}{M_{\text {outer }}}+\sum_{i=1}^{\mathrm{N}_{\mathrm{p}}-1} \mathcal{P}^{i / 3}\right)\right]^{-1}
\end{aligned}
$$

So to produce a given USP planet, the required eccentricities and inclinations are lower if we assume more outer planets which are widely spaced and have higher masses.

In Figure 4 we show the minimum $\left(e_{\mathrm{rms}}^{2}+i_{\mathrm{rms}}^{2}\right)^{1 / 2}$ from Equation (7) for $N_{p}=\{4,5,6\}$ as a function of the mass ratio $M_{\text {outer }} / M_{\mathrm{USP}}$ and the period ratio $\mathcal{P}$. We observe that for $M_{\text {outer }} / M_{\mathrm{USP}} \sim 1$, the required eccentricities and inclinations are relatively large with 
$\left(e_{\mathrm{rms}}^{2}+i_{\mathrm{rms}}^{2}\right)^{1 / 2} \sim 0.2-0.3$ for $\mathcal{P} \lesssim 5$. In turn, by increasing the mass ratio to $M_{\text {outer }} / M_{\mathrm{USP}} \sim 10$ we get $\left(e_{\mathrm{rms}}^{2}+i_{\mathrm{rms}}^{2}\right)^{1 / 2} \lesssim 0.1$, or $e_{\mathrm{rms}}, i_{\mathrm{rms}} \lesssim 0.07$ in case of equipartition $\left(e_{\mathrm{rms}} \simeq i_{\mathrm{rms}}\right)$.

For reference, there are a few systems where the USP planet has a mass measurement and there is an outer planet with a mass constraint. Kepler-10 has $M_{\text {outer }} / M_{\text {UsP }} \sim 4$ (Dumusque et al. 2014, Weiss et al. 2016), while Kepler-407b (likely $\sim 1 M_{\oplus}$ ) has a likely non-transiting outer giant planet (Marcy et al. 2014). Other non-Kepler systems include 55 Cancri with $M_{\text {outer }} / M_{\text {USP }} \sim 100$ Nelson et al. (2014) and CoRoT-7 with $M_{\text {outer }} / M_{\text {UsP }} \sim 2$ (Queloz et al. 2009). We caution that these systems might not be representative of the whole sample as there is a bias towards detecting the most massive planets from radial velocity measurements.

In conclusion, based on the conservation of angular momentum deficit, the formation of an USP planet by secular chaos roughly requires eccentricity and inclination dispersions at the level of $\left(e_{\mathrm{rms}}^{2}+i_{\mathrm{rms}}^{2}\right)^{1 / 2} \sim 0.1$ for systems with $N_{p} \gtrsim 4$ super-Earths mass planets (USP planets have $\sim$ Earth masses). For Jupiter-mass planets the required dispersions can be much lower. We shall confirm this result with numerical experiments in 4 .

\subsection{Secular excitation vs apsidal precession forces}

We consider whether the diffusive growth in eccentricity for the inner planet, once secular chaos is initiated, can be stalled by other precessional forces. This can limit the maximum eccentricity the planet can reach and prevent USP planet formation. The shortest timescale at which its pericenter distance $r_{\mathrm{p}}=a_{1}\left(1-e_{1}\right)$ is forced to vary is given by the quadrupole forcing from the closest outer companion (planet 2) and is

$$
\begin{aligned}
\tau_{\text {peri }} \equiv & \left|\frac{r_{\mathrm{p}}}{\dot{r}_{\mathrm{p}}}\right| \sim P_{1} \sqrt{1-e_{1}^{2}}\left(\frac{a_{2}}{a_{1}}\right)^{3}\left(\frac{M_{s}}{M_{2}}\right) \\
\simeq & 3.7 \times 10^{3} \mathrm{yr}\left(\frac{0.1 \mathrm{AU}}{a_{1}}\right)^{2}\left(\frac{a_{1, \mathrm{f}}}{0.02 \mathrm{AU}}\right)^{1 / 2} \\
& \times\left(\frac{15 M_{\oplus}}{M_{2}}\right)\left(\frac{a_{2}}{0.24 \mathrm{AU}}\right)^{3}
\end{aligned}
$$

where $a_{1}$ is the starting distance for the USP planet and we have evaluated the fiducial values of $M_{2}$ and $a_{2}$ as those for Kepler-10c (Weiss et al. 2016). If the planet reaches $e_{1} \sim 1$, but its inclination is still moderate $(\lesssim$ $40^{\circ}$ ) then the quadrupole forcing vanishes and $\tau_{p}$ has a longer (octupole) timescale by a factor of $\sim a_{2} /\left(e_{2} a_{1}\right)$.

\subsubsection{Relativistic precession}

The relativistic precession can change the argument of periapsis in a characteristic timescale given by

$$
\begin{aligned}
\tau_{\mathrm{GR}} & =\frac{c^{2} a_{1}\left(1-e_{1}^{2}\right)}{3 G M_{s}} P_{1} \\
& \simeq 2 \times 10^{4} \mathrm{yr}\left(\frac{a_{1}}{0.1 \mathrm{AU}}\right)^{3 / 2}\left(\frac{a_{1, \mathrm{f}}}{0.02 \mathrm{AU}}\right) .
\end{aligned}
$$

As the eccentricity increases and $a_{1, \mathrm{f}}=a_{1}\left(1-e^{2}\right)$ decreases, the above apsidal precession rate rises, leading to a quenching of the secular perturbations from the outer planet when $\tau_{\text {peri }}=\tau_{\mathrm{GR}}$, which occurs at

$a_{\mathrm{f}, \mathrm{GR}} \sim 7 \times 10^{-4} \mathrm{AU}\left(\frac{0.1 \mathrm{AU}}{a_{1}}\right)^{7}\left(\frac{15 M_{\oplus}}{M_{2}}\right)\left(\frac{a_{2}}{0.24 \mathrm{AU}}\right)^{6}$.

For instance, for Kepler-10b to reach its current semimajor axis $a_{1, f} \simeq 0.0167 \mathrm{AU}$, it should have started migration from $a_{1} \gtrsim 0.06 \mathrm{AU}\left(P_{1} \gtrsim 5.4\right.$ days $)$, which we determine by setting $0.0167 \mathrm{AU}>a_{\mathrm{f}, \mathrm{GR}}$.

\subsubsection{Precession by tidal bulges}

The tidal deformation of both the planet and the star by their mutual gravitational perturbations leads to apsidal precession. Assuming that $e_{1} \sim 1$, the characteristic timescale for the tidal bulge on the planet is given by (Sterne 1939)

$$
\begin{aligned}
\tau_{\text {tide }, \mathrm{p}} \simeq & \frac{16}{315 k_{L, p}} P_{1}\left(\frac{M_{1}}{M_{s}}\right)\left(\frac{a_{1, \mathrm{f}}}{R_{1}}\right)^{5} \\
\simeq & 4 \times 10^{5} \mathrm{yr}\left(\frac{0.3}{k_{p}}\right)\left(\frac{a_{1}}{0.1 \mathrm{AU}}\right)^{3 / 2}\left(\frac{M_{1}}{1 M_{\oplus}}\right) \\
& \left(\frac{1 R_{\oplus}}{R_{1}}\right)^{5}\left(\frac{0.02 \mathrm{AU}}{a_{1, \mathrm{f}}}\right)^{5},
\end{aligned}
$$

where $k_{L, p}$ is the tidal Love number and we scale it by that of the Earth. Similarly, the tidal bulge on the star gives rise to

$$
\begin{aligned}
\tau_{\text {tide }, \mathrm{s}} \simeq & \frac{16}{315 k_{L, s}} P_{1}\left(\frac{M_{s}}{M_{1}}\right)\left(\frac{a_{1, \mathrm{f}}}{R_{s}}\right)^{5} \\
\simeq & 6 \times 10^{7} \mathrm{yr}\left(\frac{0.014}{k_{s}}\right)\left(\frac{a_{1}}{0.1 \mathrm{AU}}\right)^{3 / 2}\left(\frac{1 M_{\oplus}}{M_{1}}\right) \\
& \left(\frac{R_{s}}{1 R_{\odot}}\right)^{5}\left(\frac{0.02 \mathrm{AU}}{a_{1, \mathrm{f}}}\right)^{5},
\end{aligned}
$$

where $k_{L, s}$ is the tidal Love number of the star and we scale it by the solar value. Thus, the precession rate is generally dominated by the tidal bulge on the planet instead of the star. As argued by Liu et al. (2015), the maximum eccentricity allowed for the tidal bulges in three-body interactions is reached when $\tau_{\text {tide,p }} \sim 0.1 \tau_{\text {peri }}$, which occurs at

$$
\begin{gathered}
a_{\mathrm{f}, \mathrm{tide}} \sim 4 \times 10^{-3} \mathrm{AU}\left(\frac{R_{1}}{1 R_{\oplus}}\right)^{10 / 9}\left(\frac{0.1 \mathrm{AU}}{a_{1}}\right)^{7 / 9} \\
\left(\frac{a_{2}}{0.24 \mathrm{AU}}\right)^{2 / 3}\left(\frac{1 M_{\oplus}}{M_{1}} \cdot \frac{15 M_{\oplus}}{M_{2}}\right)^{2 / 9} .
\end{gathered}
$$

This expression sets the minimum $a_{1}\left(1-e_{1}^{2}\right)$ allowed by tidal bulges. We note that for Kepler-10b with $R_{p} \simeq 1.46 R_{\oplus}$ and $M_{1} \simeq 3.3 M_{\oplus}$, the current location implies that migration should have started from $a_{1} \gtrsim 0.045$ AU ( $P_{1} \gtrsim 3.5$ days $)$, comparable to that obtained from relativistic precession.

\subsection{Tidal captures}

We assess whether the USP planets can attain their current detached orbits by tidal decay and whether these can prevent their tidal disruptions. We describe the roles of equilibrium tides and dynamical tides separately. 


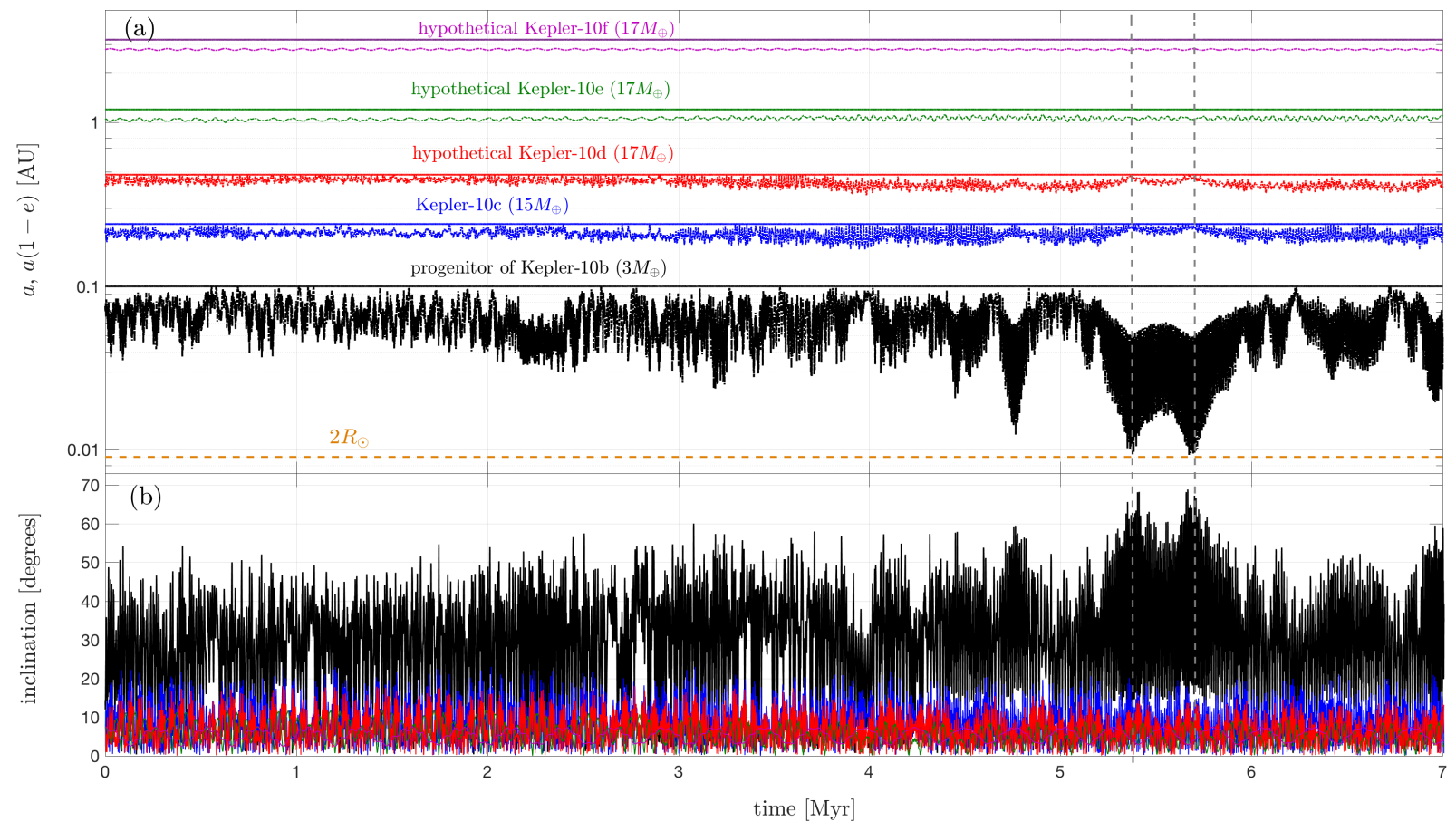

FIG. 5.- Example of a possible evolution to produce the Kepler-10 system. The upper panel (a) depicts planet semi-major axis, and pericenter distance $\left(a_{i}\left[1-e_{i}\right]\right)$, while the lower panel that of inclinations. Due to secular chaos with the companions, planet Kepler-10b reaches $a_{1}\left(1-e_{1}^{2}\right) \lesssim 0.02 \mathrm{AU}$ after $\sim 5 \mathrm{Myr}$, at which time tidal damping ( $f$-mode diffusion or equilibrium tides) can potentially shrink the orbit to a final period $\leqslant 1$ day. At the moment that the planet reaches its maximum eccentricity (indicated by gray vertical lines), its inclination is also the highest $\left(i \sim 70^{\circ}\right)$, while the eccentricities of planets $\mathrm{c}$ and $\mathrm{d}$ are low $(\lesssim 0.05)$. The initial eccentricities and inclinations for planets $\mathrm{c}$ to $\mathrm{f}$ are assumed to be $e=0.12$ and $i=7^{\circ}$, while higher values $\left(e_{1}=0.22\right.$ and $\left.i_{1}=12^{\circ}\right)$ are assumed for Kepler-10b to speed up its chaotic diffusion.

\subsubsection{Equilibrium tides}

Since the planet's spin synchronizes with the orbit in short timescales compared with migration timescales, the planet needs to be in an eccentric orbit for friction to extract orbital energy.

We describe the tidal effects on the orbital evolution of the planet using the weak friction theory of equilibrium tides (e.g., Hut 1981), according to which the rate of decay of the semi-major axis for a pseudo-synchronized planet can be written as

$$
\begin{aligned}
\left(\tau_{a}^{\text {planet }}\right)^{-1} \equiv & \left|\frac{a_{1}}{a_{1}}\right|=6 k_{L, p} \tau_{p}\left(\frac{G M_{s}}{a_{1, \mathrm{f}}^{3}}\right)\left(\frac{R_{1}}{a_{\mathrm{f}}}\right)^{5} \\
& \times \sqrt{\frac{a_{1, \mathrm{f}}}{a_{1}}} \frac{M_{s}}{M_{1}} \mathcal{F}\left(e_{1}\right),
\end{aligned}
$$

where $a_{1, \mathrm{f}}=a_{1}\left(1-e_{1}^{2}\right)$ is the final circularization radius, $k_{L, p}$ is the tidal Love number, $\tau_{p}$ is the tidal lag time (assumed constant in the weak friction theory), and

$$
\begin{aligned}
\mathcal{F}(e)= & +\frac{31}{2} e^{2}+\frac{255}{8} e^{4}+\frac{185}{16} e^{6}+\frac{25}{64} e^{8} \\
& -\frac{\left(1+\frac{15}{2} e^{2}+\frac{45}{8} e^{4}+\frac{5}{16} e^{6}\right)^{2}}{1+3 e^{2}+\frac{3}{8} e^{4}} .
\end{aligned}
$$

From Equation (14) the timescale to form an USP planet with final period $P_{1, \mathrm{f}}=1$ days $\left(a_{1, \mathrm{f}}=a_{1}[1-\right.$ $\left.\left.e_{1}^{2}\right] \simeq 0.02 \mathrm{AU}\right)$ starting from a period $P_{1}$ around a Sun- like star becomes

$$
\begin{aligned}
\tau_{a}^{\text {planet }} \simeq & 1.37 \times 10^{3} \text { yr } \frac{1}{k_{L, p} \tau n_{1, \mathrm{f}}}\left(\frac{1 R_{\oplus}}{R_{1}}\right)^{5}\left(\frac{M_{1}}{1 M_{\oplus}}\right) \\
& \times\left(\frac{P_{1, \mathrm{f}}}{1 \text { day }}\right)^{4}\left(\frac{P_{1}}{10 \text { day }}\right)^{1 / 3}\left(\frac{\mathcal{F}(0.9)}{\mathcal{F}\left(e_{1}\right)}\right) \\
\simeq & 10^{5} \mathrm{yr}\left(\frac{0.3}{k_{L, p}}\right)\left(\frac{600 \mathrm{~s}}{\tau_{p}}\right)\left(\frac{1 R_{\oplus}}{R_{1}}\right)^{5}\left(\frac{M_{1}}{1 M_{\oplus}}\right) \\
& \times\left(\frac{P_{1, \mathrm{f}}}{1 \text { day }}\right)^{5}\left(\frac{P_{1}}{10 \text { day }}\right)^{1 / 3}\left(\frac{\mathcal{F}(0.9)}{\mathcal{F}\left(e_{1}\right)}\right)
\end{aligned}
$$

For Earth-like planets we assume $\tau_{p} \sim 600 \mathrm{~s}$ (Lambeck 1977, Neron de Surgy \& Laskar 1997).

Using the tidal quality factor $Q_{p} \equiv\left(\tau_{p} \omega\right)^{-1}$ with $\omega=$ $n_{1, \mathrm{f}} \equiv\left(G M_{s} / a_{1, \mathrm{f}}^{3}\right)^{1 / 2}$ we obtain

$$
\begin{aligned}
\tau_{a}^{\text {planet }} \simeq & 1.2 \times 10^{5} \text { yr }\left(\frac{Q_{p} / k_{L, p}}{100}\right)\left(\frac{1 R_{\oplus}}{R_{1}}\right)^{5}\left(\frac{M_{1}}{1 M_{\oplus}}\right) \\
& \times\left(\frac{P_{1, \mathrm{f}}}{1 \text { day }}\right)^{4}\left(\frac{P_{1}}{10 \text { day }}\right)^{1 / 3}\left(\frac{\mathcal{F}(0.9)}{\mathcal{F}\left(e_{1}\right)}\right) .
\end{aligned}
$$

Evidently this timescale is short enough so that if planets can attain eccentricities large enough to reach $P_{1, \mathrm{f}}=$ $P_{1}\left(1-e_{1}^{2}\right)^{2 / 3} \lesssim 1$ day $\left(a_{1 \mathrm{mf}} \lesssim 0.02 \mathrm{AU}\right)$, then circularization is possible. However, since relativistic precession and tidal bulges do not efficiently limit the eccentricity growth, the pericenter can continue shrinking until the planet gets tidally disrupted. 
The disruption can be prevented by a tidal capture, meaning that the planet can shrink its orbit significantly by tidal dissipation before the pericenter continues to approach the disruption distance. This possibility seems promising because, as shown by Muñoz et al. (2016), rocky planets can survive secular migration for a wide range of parameters compared to gaseous planets.

By equating $\tau_{\text {peri }}$ (Eq. [8]) and $\tau_{a}^{\text {planet }}$ (Eq. [17]) we get that a tidal capture occurs at

$$
\begin{aligned}
a_{\mathrm{f}, \text { capture }} \simeq & 0.011 \mathrm{AU}\left(\frac{R_{1}}{1 R_{\oplus}}\right)^{10 / 11}\left(\frac{a_{2}}{0.24 \mathrm{AU}}\right)^{6 / 11} \\
& \left(\frac{0.1 \mathrm{AU}}{a_{1}}\right)^{5 / 11}\left(\frac{100}{Q_{p} / k_{L, p}} \cdot \frac{1 M_{\oplus}}{M_{1}} \cdot \frac{15 M_{\oplus}}{M_{2}}\right)^{2 / 11}(18)
\end{aligned}
$$

For Kepler-10b with $R_{1}=1.47 R_{\oplus}$ we get the following condition for a capture at its current location (i.e., $\left.a_{\mathrm{f}, \text { capture }}=0.0167 \mathrm{AU}\right)$

$$
\left(\frac{a_{1}}{0.1 \mathrm{AU}}\right)^{5 / 11}\left(\frac{Q_{p} / k_{L, p}}{100}\right)^{2 / 11} \simeq 0.56
$$

Thus, it is possible that Kepler-10b has achieved its current location by a tidal capture if it started migration from $a_{1} \sim 0.06 \mathrm{AU}$.

We stress that the final semi-major achieved by a tidal capture is the minimum value allowed by tidal dissipation in the planet. The actual value for a planet undergoing high-eccentricity migration might be longer for the following separate reasons:

1. tidal dissipation shrinks the orbit after several secular cycles, not one as it is assumed in a tidal capture;

2. the secular forcing can have a longer timescale than the one used above ( $\tau_{\text {peri }}$ in Eq. 8]) because it can be driven by the octupole moment, not the quadrupole, from the outer planetary orbit. If so, $\tau_{\text {peri }}$ increases by a factor of $\sim a_{2} /\left(e_{2} a_{1}\right)$, increasing $a_{\mathrm{f}, \text { capture }}$ by a factor of $\sim\left[a_{2} /\left(e_{2} a_{1}\right)\right]^{2 / 11}$.

\subsubsection{Dynamical tides: diffusive $f$-mode excitation preventing disruptions}

As discussed above, the tidal dissipation rate in the planet from equilibrium tides might be efficient enough to tidally capture the proto-USP planet and prevent its disruption. However, the dissipative properties of the short-period planets are quite uncertain, and the values of $Q_{p} / k_{L, p}$ can potentially be large enough that they invalidate our previous statement (e.g., GJ 876-d, $\mathrm{Pu}-$ ranam \& Batygin 2018).

Fortunately, even in the limit of an inviscid planet $\left(Q_{p} \rightarrow \infty\right)$, there is salvation. It was recently pointed out by Vick \& Lai (2018) and Wu (2018) that, regardless of the planetary dissipative properties, a planet in a highly eccentric orbit can diffusively excite its spherical-degree 2 fundamental mode (f-mode) to near-unity amplitudes. When this happens, that nonlinear effects can set in and effectively convert mode energy to heat. As a result, the orbital energy is lost and the orbit shrinks in a short timescale. This circumvents the difficulty surrounding the uncertain dissipation of the equilibrium tide.
As shown by $\mathrm{Wu}(2018)$, the mode excitation can enter the diffusive regime and the mode energy can grow linearly in time when the pericenter distance $r_{p}=a_{1}\left(1-e_{1}\right)$ reaches

$$
r_{p} \lesssim 4 R_{p}\left(\frac{M_{s}}{M_{1}}\right)^{1 / 3} \simeq 0.012 \mathrm{AU}\left(\frac{5 \mathrm{~g} / \mathrm{cm}^{3}}{\rho}\right)^{1 / 3},
$$

with some small corrections that depends on the $f$-mode period. Here $\rho$ is the density of the planet. At this point, the orbit shrinks with a rapid timescale of (Eq. [17] in $\mathrm{Wu}$ 2018)

$$
\tau_{a, \mathrm{f} \text {-mode }} \sim 10^{2} \mathrm{yr}\left(\frac{a_{1}}{0.1 \mathrm{AU}}\right)^{3} .
$$

Equation (20) also yields the final orbital semi-major axis by $a_{1, f} \approx 2 r_{p}$. This is because $r_{p}$ also constitutes an impassable wall for the planet migration. The timescale for mode growth drops steeply as $r_{p}^{-21}$, any further decrease of the pericenter distance beyond that in Equation (20) brings exponentially faster decay in orbital energy. This efficiently decouples the planet from its secular perturber. When the planet's orbit circularizes from $e_{1} \approx 1$, we obtain $a_{1, f} \approx 2 r_{p}$, or

$a_{\mathrm{f}, \mathrm{f}-\text { mode }} \lesssim 8 R_{p}\left(\frac{M_{s}}{M_{1}}\right)^{1 / 3} \simeq 0.024 \mathrm{AU}\left(\frac{5 \mathrm{~g} / \mathrm{cm}^{3}}{\rho_{p}}\right)^{1 / 3}(22)$

which corresponds to a final period $\lesssim 1.3$ days.

In conclusion, the diffusive excitation of the $f$-mode allows for rapid orbital migration when the planet reaches inwards of the value in Equation (20). This prevents any secularly migrating planets from being pushed even closer to the star and suffering the fate of tidal disruption. We end up with an USP planet that lies around the observed distances, subject to uncertainties in f-mode period, planet density, etc. Subsequent tidal dissipation, possibly via equilibrium tides (likely for solid planets), may eventually circularize the orbit.

\section{NUMERICAL EXPERIMENTS}

We explore numerically the validity of our analytical estimates in the previous section by running direct numerical integrations of a possible initial configuration of the Kepler-10 system. Our calculations should be taken as a proof of concept and not as a detailed population synthesis study, which is beyond the scope of this work.

\subsection{Code}

All integrations were performed using the WHFAST integrator (Rein \& Tamayo 2015) in the open-source REBOUND N-body package (Rein \& Liu 2011). We include the effects from relativistic precession and apsidal precession from tidal bulges from the REBOUNDx ${ }^{5}$ library with the option gr-potential and Love numbers $k_{L, p}=0.3$ and $k_{L, s}=0.014$ for the planets and the star, respectively (Tamayo et al., in prep.).

Our experiments do not include tidal dissipation and we use the maximum eccentricity $e_{1, \max }$ as a proxy for the potential formation of an USP planet: planets reaching $a_{1}\left(1-e_{1, \max }^{2}\right) \lesssim 0.02 \mathrm{AU}$ can be tidally captured to a final semi-major axis of $\lesssim 0.02 \mathrm{AU}\left(P_{1} \lesssim 1\right.$ day $)$.

\footnotetext{
5 https://github.com/dtamayo/reboundx
} 


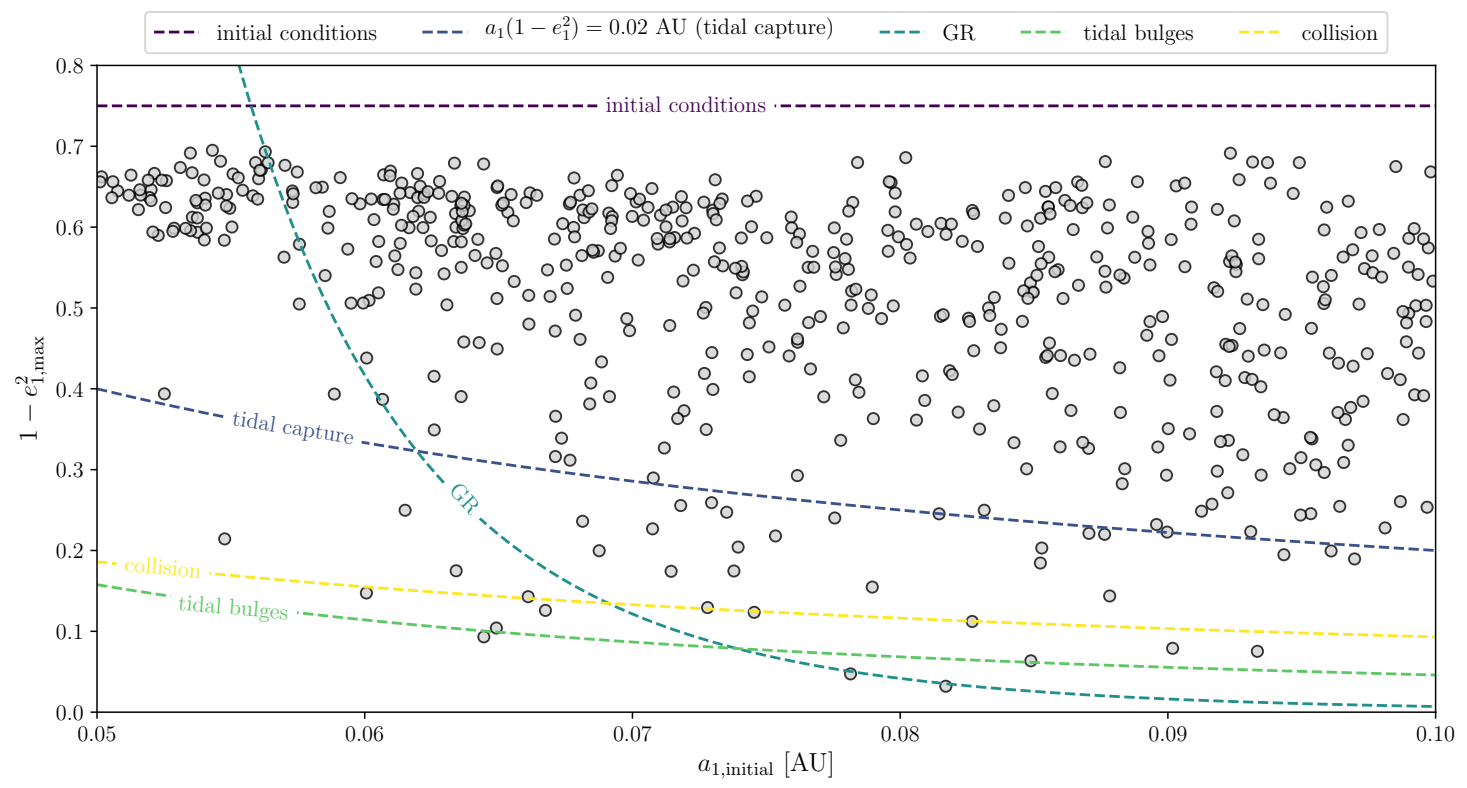

FIG. 6. - Maximum eccentricities reached by a Kepler-10b-like planet after $10^{6} \mathrm{yr}$ for different initial semi-major axes. We place planet b with an initial high eccentricity $\left(e_{1}=0.5\right.$, purple horizontal line at 0.75$)$ and inclination $\left(i_{1}=28.6^{\circ}\right)$ to artificially speed up the diffusion to large $e_{1}$. The outer planets have eccentricities and inclinations drawn from a Rayleigh distribution with $\sigma_{e}=\sigma_{i}=0.1$ (rms values of $0.14)$ and have the same spacing and masses as in Figure 5 The remaining orbital elements $(f, \omega, \Omega)$ are drawn randomly. The integrations include relativistic precession and non-dissipative tidal bulges, and we indicate the maximum eccentricities allowed by relativistic precession (Eq. 10]) and tidal bulges (Eq. [12]). For reference we indicate the threshold to become an USP planet of $a_{1}\left(1-e_{1}^{2}\right)<0.02$ AU. The integration timestep is 0.1 day.

\subsection{Example and orbital architecture}

We shall assume that the Kepler-10 system has planets beyond $\sim 100$ days and that these planets have Neptune masses, similar to the mass of Kepler-10c (Weiss et al. 2016). We place these hypothetical Kepler-10x planets with periods $\simeq 122$ days, $\simeq 480$ days, and $\simeq 2100$ days. Although our choice of orbital configurations is arbitrary, its general architecture is broadly consistent with the bulk of planetary systems in the Kepler sample:

- there are three planets inside $\sim 400$ days, consistent with the average number of planets in this rang $\biguplus^{6}$ (Zhu et al. 2018);

- the Neptune-size planets in the range of $\sim 2-25$ years are at least as common than their sub-year period counterparts (Foreman-Mackey et al. 2016).

In Figure 5, we show the evolution of one possible progenitor of the Kepler-10 system. Here, Kepler-10b starts at $a_{1}=0.1 \mathrm{AU}$ and its eccentricity and inclination evolve to large values due to secular chaotic diffusion driven by the outer planets (e.g., Laskar 1996: Wu \& Lithwick 2011). As expected from these secular perturbations, the semi-major axes of all the planets remain constant (indicated by the horizontal solid lines).

The planet b reaches a maximum eccentricity of $e_{1} \simeq$ 0.9 after $\sim 5 \mathrm{Myr}$, so its pericenter distance becomes $a_{1}\left(1-e_{1}\right) \simeq 2 R_{\odot}$ and it could be tidally captured to become an USP planet $\left(a_{1}\left[1-e_{1}^{2}\right] \lesssim 0.02 \mathrm{AU}\right)$. At this point (indicated by vertical dashed lines) its inclination is also largest $\left(i_{1} \sim 70^{\circ}\right)$ so if the planet were tidally

${ }^{6}$ Planet Kepler-10c has TTVs, indicating the presence of a third and unseen planet in the system (Weiss et al. 2016). captured, it would likely have a large inclination relative to the outer planets and the host star spin axis.

In our picture, secular chaos is a means to stabilize the system by reducing its overall angular momentum deficit (Wu \& Lithwick 2011). If planet Kepler-10b were tidally captured at $e_{1, \max }$, then the orbits of the outer planets would gain angular momentum and become more circular. In fact, at $e_{1, \max }$ (vertical dashed lines) the planets $\mathrm{c}$ and $\mathrm{d}$ have eccentricities of $e \simeq 0.05$ compared to their averages of $\sim 0.15$ during the rest of the evolution.

\subsection{Maximum eccentricities allowed by short-range forces}

In Figure 6 we show the maximum eccentricities reached for 500 integrations with the same architecture as Figure 5 but changing the initial semi-major axis of planet $b$ to illustrate the effect from short-range forces and compare with our analytical estimates from $\$ 3.2$.

We initialize the orbit of the innermost planet with $e_{1}=i_{1}=0.5$ to artificially speed up the diffusion to large eccentricities as we use a short integration timestep (0.1 day) and relatively small maximum integration time (1 Myr). Our goal is to properly resolve very large eccentricities $\left(e_{1} \gtrsim 0.9\right)$. The subsequent experiments do not assume initial large $e, i$ for the innermost planet.

We observe that the distribution of $1-e_{1, \max }^{2}$ looks roughly uniform for systems with initial semi-major axes $\gtrsim 0.075 \mathrm{AU}$, while it significantly shrinks towards the initial values $\left(1-e_{1}^{2}=0.75\right)$ for $\lesssim 0.06 \mathrm{AU}$ as expected from the short-range forces.

From $\$ 3.2$ (Eqs. 10]) and (Eq. 12]) we expect that tidal bulges limit the maximum eccentricity for $a_{1} \gtrsim 0.07$ $\mathrm{AU}$, and we observe that apart from a few exceptions, the planets are indeed above the line from the tidal bulges 
(green lines). For $a_{1} \lesssim 0.07 \mathrm{AU}$, relativistic precession is expected to dominate and the bulk of the experiments are roughly above this boundary.

Finally, we notice that both relativistic precession and tidal bulges allow for the formation of USP planets for $a_{1} \gtrsim 0.06 \mathrm{AU}$ : the line at $a_{1}\left(1-e_{1}^{2}\right)=0.02 \mathrm{AU}$ is above $\mathrm{GR}$ and tides in this semi-major axis range.

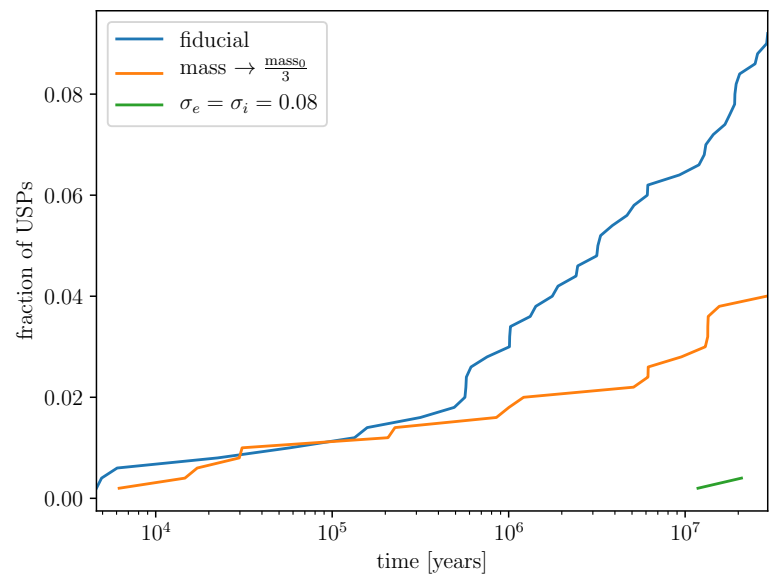

FIG. 7. - Fraction of USP planets formed (i.e., reaching $a_{1}(1-$ $\left.e_{1}^{2}\right)<0.02 \mathrm{AU}$ ) as a function of time for Kepler-10 systems where the innermost planet starts with a random semi-major axis in $a_{1} \in$ $[0.06,0.1]$ AU. In our fiducial run (blue line), all planets have initial eccentricities and inclinations drawn from a Rayleigh distribution with parameter $\sigma=0.1\left(e_{\mathrm{rms}}=i_{\mathrm{rms}}=0.14\right)$ and the masses and semi-major axes of the outer planets are the same as in Figure 5 The green line indicates a simulation in which we decrease the dispersions by setting $\sigma=0.08\left(e_{\mathrm{rms}}=i_{\mathrm{rms}}=0.11\right) ; 2$ out 500 become USP planets, while the orange line shows a set where we decrease the masses of the planets by a factor of $3\left(M_{\text {outer }} \sim 5 M_{\oplus}\right)$. The integrations include GR precession and non-dissipative tidal bulges.

\subsection{Formation rate of USP planets}

We run 500 experiments similar to those in Figure 6 . but for longer timescales of $30 \mathrm{Myr}$ and drawing the eccentricities and inclinations of all planets from a Rayleigh distribution with parameter $\sigma=0.1\left(e_{\mathrm{rms}}=i_{\mathrm{rms}}=\right.$ 0.14). We set the integration time-step to 1 day ${ }^{7}$ and stop the simulation when $a_{1}\left(1-e_{1}^{2}\right)<0.02$ AU (happening typically at $\left.e_{1} \sim 0.8-0.9\right)$, at which time we assume the planet is tidally captured (Eq. [22]). This is our fiducial set of integrations. Our goal is to get an estimate of the fraction of systems that can become USP planets and how it depends on the evolution timescale.

In Figure 7 we show the fraction of USP planets as a function of time (solid blue lines). Recall that we take the condition $a_{1}\left(1-e_{1}^{2}\right)<0.02 \mathrm{AU}$ as a proxy for tidal capture. We observe that in these integrations the fraction reaches up to $46 / 500 \simeq 9 \%$ with most systems reaching the tidal capture threshold after $\sim 1$ Myr $(\sim 100$ to

7 This time-step is somewhat large and barely resolves the pericenter passages when the planet becomes an USP planet $\left(a_{1}\left[1-e_{1}^{2}\right] \simeq 0.02 \mathrm{AU}\right)$. However, we checked that by decreasing the time-step to 0.5 days for up to 10 Myr we get consistent results for the fraction of USP planets. We also checked that the secular code Rings, downloadable at https://github.com/farr/Rings gives consistent results.
$10^{4}$ secular cycles, Eq. 8). The fraction of USP planets does not level off after $30 \mathrm{Myr}\left(\sim 10^{9} P_{1}\right)$ and longer integrations are required to assess whether the ensemble reaches a saturation state. We can only say at least $\simeq 9 \%$ of these Kepler-10-like systems can produce USP planets. We briefly study how this fraction depends on the eccentricity and inclination dispersions as well as the planetary masses.

Lowering eccentricity and inclination dispersions- We have repeated our fiducial integrations but reduced the eccentricity and inclination dispersions slightly by changing the parameter from $\sigma=0.1\left(e_{\mathrm{rms}}=i_{\mathrm{rms}}=0.11\right)$ to $\sigma=0.08\left(e_{\mathrm{rms}}=i_{\mathrm{rms}}=0.11\right.$; green line $)$ and observe that the final fraction of USP planets decreases from $\simeq 9 \%$ to $2 / 500=0.4 \%$. We have checked that the systems that undergo fast eccentricity diffusion and become USP planet within $30 \mathrm{Myr}$ are those that have the largest initial values of AMD (or $\sum\left[e_{i}^{2}+i_{i}^{2}\right]$; Eq. 4 ). Thus, by slightly reducing $\sigma$ by $20 \%$ we reduce the expected AMD by the same factor. In order to asses whether smaller values of $\sigma$ allow for significant formation of USP planets we need to integrate these systems for Gyr timescales $\left(10^{11} P_{1}\right)$. This study is beyond the scope of our paper and it should be the topic of a separate work.

Lowering planetary masses - We have repeated our fiducial integrations but decreased the masses of all the planets by a factor of 3 (orange lines), so the masses might be more representative of the overall Kepler sample with $M_{\mathrm{USP}} \sim 1 M_{\oplus}$ and $M_{\text {outer }} \sim 5 M_{\oplus}$. The fraction of USP planets decreases from $\simeq 9 \%$ in the fiducial simulation to $20 / 500 \simeq 4 \%$. This decrease is expected because the planetary mass scale affects the timescale of the secular evolution $\left(\tau_{\mathrm{sec}} \propto 1 / M_{\mathrm{p}}\right)$. Thus, the fraction of USP planets in the runs with 3 times lower masses up to $30 \mathrm{Myr}$ of $\simeq 4 \%$ should be compared to the fraction in the fiducial simulation up to $10 \mathrm{Myr}$ (i.e., fixed $t_{\max } / \tau_{\mathrm{sec}}$ ), which corresponds to $\simeq 6 \%$. The small difference (not statistically significant) between these fractions seems to be due to relativistic precession since it suppresses the diffusion to large eccentricities more efficiently in systems with lower planetary masses.

\subsection{Inclinations of USP planets}

In Figure 8 we show the inclinations of the planets that can become USP planets at the moment they first reach $a_{1}\left(1-e_{1}^{2}\right)<0.02 \mathrm{AU}$ and would get tidally capture in our fiducial integrations (orange lines). These inclinations extend from $\sim 5^{\circ}$ to $\sim 50^{\circ}$, and in one third of the cases these reach above $\sim 30^{\circ}$ and are substantially higher than the initial distribution with a median of $\simeq 6.7^{\circ}$ (Rayleigh with $\sigma=0.1$ ).

\subsection{Non-migrating planets: eccentricities and inclinations}

In Figure 8 we compute the time-average inclination of the systems that do not form USP planets (blue lines). These systems have an initial distribution with a median of $\simeq 6.7^{\circ}$ (Rayleigh with $\sigma=0.1$ ) that broadens and reaches a median of $\simeq 12^{\circ}$ as a result of the secular excitation. This implies that the secular gravitational interactions broadens the inclinations of the innermost planets significantly. 


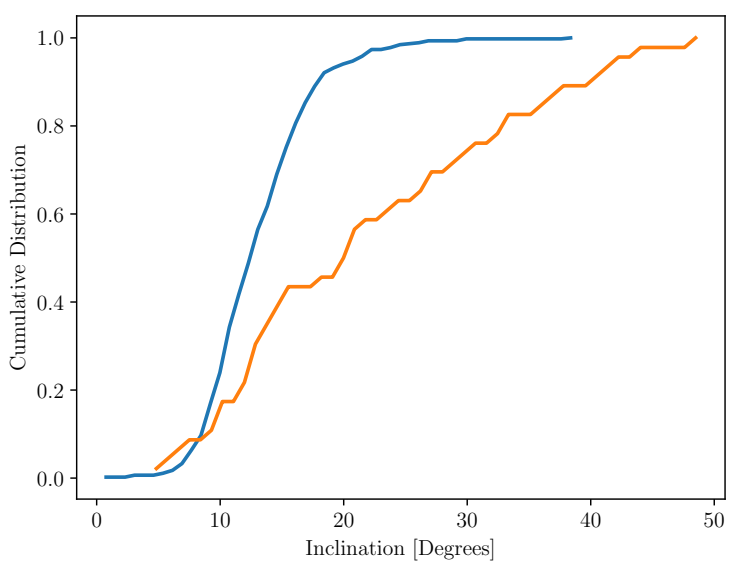

FIG. 8. - Inclination distributions for Kepler-10b from our fiducial simulation (initial $e, i$ from Rayleigh with $\sigma=0.1$, blue line in Figure 7]. The blue line indicates the distribution of the timeaveraged inclination for the systems that do not form USP planets, while the orange line indicates the inclinations of systems when they first reach $a_{1}\left(1-e_{1}^{2}\right)<0.02 \mathrm{AU}$ and would be tidally captured.

Similarly, in Figure 9 we show the time-averaged eccentricity (red line) for the systems without USP planets and compare this with the initial distribution (black line, Rayleigh with $\sigma=0.1$ ). As it happens with the inclinations, the eccentricities broaden significantly from an initial median of $\simeq 0.11$ to a time-averaged median of $\simeq 0.22$ (the red dashed line indicates a Rayleigh distribution with $\sigma=0.2$ for reference).

The results above are consistent with the idea that secular chaos can drive the system toward equipartition of energy of the different secular degrees of freedom (AMD equipartition), where $M_{i} \sqrt{a_{i}} e_{i}^{2}$ and $M_{i} \sqrt{a_{i}} i_{i}^{2}$ reach similar values for all $i$ (Wu \& Lithwick 2011). Since the innermost planet has the lowest circular angular momentum (lowest $M_{i} \sqrt{a_{i}}$ ), it gets a larger chunk of the system's eccentricity and inclination budget. In particular, since $M_{\text {outer }} / M_{\mathrm{USP}} \sim 4$ for our example based on Kepler-10, we expect a factor of $\sim 2$ increase in the time-averaged eccentricities and inclinations, consistent with the results above.

In summary, we find that secular gravitational interactions leads to excitation of the inner planet's eccentricities and inclinations from an initial Rayleigh distribution with $\sigma=0.1$ to a time-averaged distribution with $\sigma \simeq 0.2$. This result is consistent with secular chaos driving the system towards equipartition of Angular Momentum Deficit.

\section{DISCUSSION}

We propose that most of the ultra-short-period planets around FGK stars are migrated inward by the combined effects of secular chaos and tidal dissipation in the planets. These planets commence their migration from orbital periods beyond $\sim 5$ days.

The key ingredients for our proposal are: the presence of several planets in the system and a moderate amount of eccentricities and/or inclinations $\left(e_{\mathrm{rms}}, i_{\mathrm{rms}} \sim 0.1\right)$ to drive chaotic diffusion. As discussed in the introduction, this set of requirements agrees with the observed orbital architecture of planetary systems in the Kepler sample.

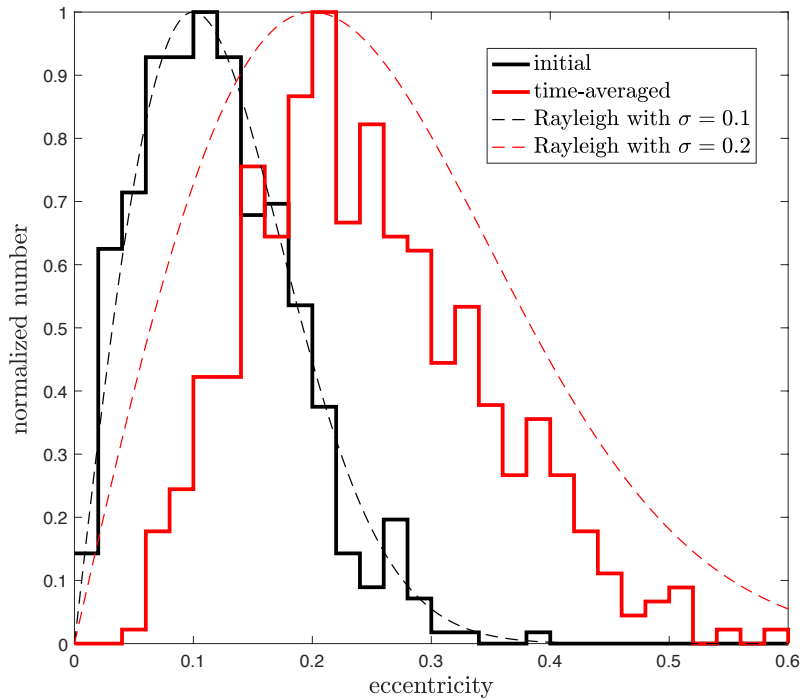

FIG. 9. - Eccentricity distributions for the Kepler-10b planets that do not reach the threshold $a_{1}\left(1-e_{1}^{2}\right)<0.02$ AU to become USP planets in our fiducial integrations ( $e, i$ from Rayleigh with $\sigma=0.1$ and $t_{\max }=30 \mathrm{Myr}$ ). The red line indicates the timeaveraged distribution of the ensemble, while the black line shows the initial distribution. For reference the thin dashed lines show the Rayleigh distributions with $\sigma=0.1$ (black) and $\sigma=0.2$ (red). The distributions are normalized by the tallest bin.

In what follows, we discuss various predictions from our model and comment on previous works on this subject.

\subsection{Properties of USP planets from secular chaos 5.1.1. Occurrence rate of USP planets}

The observed occurrence rate of USP planets around GK dwarfs is $\sim 0.5-1 \%$ (Sanchis-Ojeda et al. 2014). We consider whether our proposal can explain this rate.

The occurrence rate of Kepler systems among the same stars is $\sim 30 \%$ (Zhu et al. 2018). A fraction of these are compact, high-multiple systems that are too tight to be secularly interacting, and are instead dominated by mean-motion resonances. The likely progenitors for USP planets are widely-spaced systems that only contain three or fewer planets within 400 days. This latter is about half of the overall population (Zhu et al. 2018). For these systems, secular chaos has to produce USP planets at an efficiency of $\sim 5 \%$ to account for most of the observed USP planets.

For our fiducial planet architecture, with $e_{\mathrm{rms}}, i_{\mathrm{rms}} \sim$ 0.1 , and 4 Neptune-massed planets outside the USP planet progenitor, we find that $10 \%$ of the systems have enough AMD to both cause secular chaos and to raise the inner planet's eccentricity toward tidal capture, within our integration time of 30 Myrs. Due to the diffusive nature of secular chaos, it is expected that this fraction will grow with time, but it is hard to project where it will end up at a few Gyrs. ${ }^{8}$ In the meantime, our experiments show that dropping the AMD by some $20 \%$ (from Rayleigh with $\sigma=0.1$ to 0.08 ) sharply reduces the yield within 30 Myrs by a factor of 25, while reducing the masses for the outer companions does not sharply

8 If the linear growth (with logarithmic time) as seen in Fig. 7 continues, the final ratio will be $\sim 40 \%$. 
trim down the yield. These considerations argue that, in order for the sparsely spaced Kepler systems to produce the desired rate of USP planets, the values of eccentricity and inclination dispersion are of order 0.1 , our fiducial value.

Such a dispersion, interestingly, coincides with what is currently determined. Zhu et al. (2018) estimated that sparse systems (with three planets or fewer within 400 days) have $i_{\text {rms }} \sim 0.05-0.1$; and (Xie et al. 2016) argued that $e_{\mathrm{rms}} \gtrsim 0.1$ for these same systems, which typically appear as single-transiting planets in the Kepler database.

So we conclude that, as long as most Kepler systems contain a fair number of outer companions (as suggested by results from Foreman-Mackey et al. 2016, Suzuki et al. 2016), our mechanism is likely to account for the observed rate of USP planets.

\subsubsection{Orbital periods: why the 1-day limit for USP planets?}

The conventional definition of an USP planet as one that orbits with an orbital period $<1$ day is, in principle, arbitrary. Here we adopt this definition by arguing that there is something physical about the 1-day cut.

In our model, an USP planet is defined as a planet that gets tidally captured. The efficacy of tidal capture drops off steeply beyond a few Roche radii. For instance, tidal capture by $f$-mode excitation leads to the formation of a planet with a final period of $\lesssim 1\left(\rho_{\oplus} / \rho\right)^{1 / 3}$ day, which occurs around 1 day for planets of Earth density. So we expect planets inward of 1 day have been placed there by tidal capture, while planets outward should not have experienced this process. This naturally explain why only the USP planets are dynamically detached from the companions and why planets at $\sim 1-3$ day orbits are less so.

All this being said, it might also be possible to migrate planets to these latter distances $(1-3$ days $)$ via secular chaos. In the case that the innermost planet does not reach the distance for tidal capture but has acquired some substantial eccentricity, tidal circulation is sufficiently efficient that its orbit will decay gradually and it is eventually freed from the influences of other planets. In fact, Figure 1 shows a handful of systems with periods in $\sim 1-3$ days that have distant companions ( 5 systems have period ratios of $\gtrsim 10$ ) and for which higheccentricity might have operated. This possibility should be addressed with a full population synthesis including tidal dissipation.

\subsubsection{Stellar obliquities}

Secular chaos leads to non-linear mixing between the eccentricity and inclination modes, resulting in large excursions in eccentricities and in inclinations.

In our experiments in Figure 8 we find that the inclinations of the USP planets relative to the initial reference plane can often reach $\sim 20^{\circ}-50^{\circ}$, much larger than the initial inclination dispersion.

The inclinations reached by USP planets in these experiments are generally lower than previous experiments of secular chaos in hot Jupiter systems by Hamers et al. (2017) where planets reach a broad range in $0-140^{\circ}$ with $\sim 10 \%$ being retrograde (similar results were found by Lithwick \& Wu 2014). We believe that the main difference between the hot Jupiter set-up and ours is that

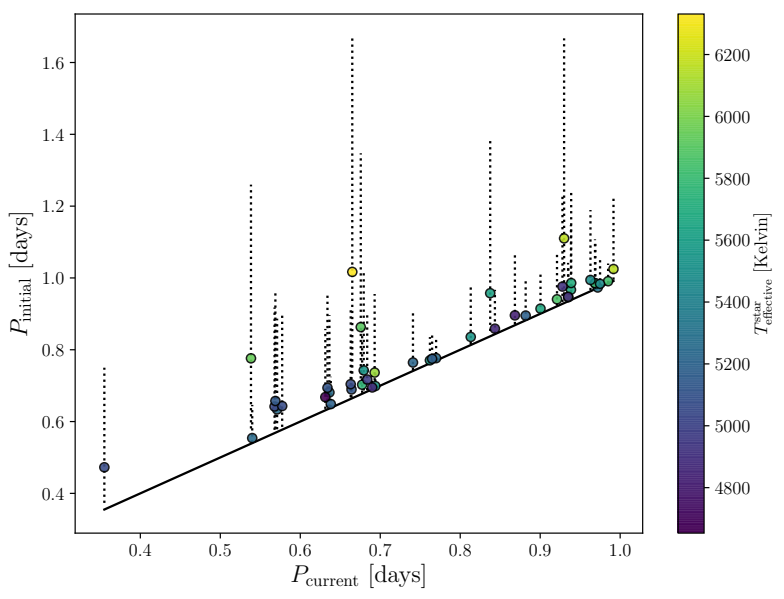

FIG. 10.- Initial period from which the USP planets started tidal migration if only asynchronous equilibrium tides raised on the star were operating and $P_{\text {spin }} \gg 1$ day. We adopt $Q_{s} / k_{L, s}=10^{7}$ and the lines indicate the range $Q_{s} / k_{L, s}=\left[10^{6}, 10^{8}\right]$, while the lifetime of each system is assumed to be 5 Gyr. The color coding indicates the effective temperature of the host stars.

the USP planets start migration much closer in, so these only need to reach $e_{1} \sim 0.9$ in order to migrate. On the other hand, hot Jupiters need to reach $e_{1} \gtrsim 0.98$ and a larger maximum eccentricity might translate into larger attainable inclinations.

\subsection{Other Formation Proposals}

We have provided comments on some proposed scenarios for USP planet formation in the introduction section. Here, we provide more detailed assessments on a couple scenario that are similar to ours and involve both dynamical perturbations by other planetary bodies and/or tidal evolution.

\subsubsection{Detaching ultra-short-period planets by tides raised on} the star

It was recently suggested by Lee \& Chiang (2017) that the orbits of close-in planets can be eroded from the inner edges of proto-planetary disks to ultra-short-periods by asynchronous tides raised on their slowly-spinning host stars. This could, in principle, also explain why USP planets are more widely spaced than their longer-period counterparts. We asses this proposal here.

The timescale to shrink its orbit by asynchronous equilibrium tides acting on the host star is (e.g., Mardling \& Lin 2002)

$$
\tau_{a}^{\text {star }} \equiv\left|\frac{a}{\dot{a}}\right|=\frac{P_{1}}{9 \pi} \frac{M_{s}}{M_{1}}\left(\frac{a_{1}}{R_{s}}\right)^{5} \frac{Q_{s}}{k_{L, s}}\left|1-\frac{P_{1}}{P_{\text {spin }}}\right|^{-1}
$$

where $Q_{s}$ and $k_{L, s}$ are tidal quality factor and the Love number of the star. For reference, for a Sun-like star with $P_{\text {spin }} \gg 1$ day one obtains

$$
\begin{aligned}
\tau_{a}^{\text {star }} \simeq & 510^{10} \text { yr }\left(\frac{P_{1}}{1 \text { day }}\right)^{13 / 3}\left(\frac{1 M_{\oplus}}{M_{1}}\right)\left(\frac{1 R_{\odot}}{R_{s}}\right)^{5} \\
& \times\left(\frac{M_{s}}{1 M_{\odot}}\right)\left(\frac{Q_{s} / k_{L, s}}{10^{6}}\right)
\end{aligned}
$$


which is an order of magnitude longer than the typical ages of the host stars and typical values of the modified quality factor constrained from observations $Q_{s} / k_{L, s} \gtrsim$ $10^{6}$ (e.g., Ogilvie 2014)

We further quantify the extent at which tides on the star can shrink the orbits of USP planets. We integrate Equation 23 backwards in time and estimate the initial period $P_{\text {initial }}$ the planets would have had in order to reach their current locations $P_{\text {current }}$ after a time $t_{\text {age }}$ :

$$
P_{1, \text { initial }}=P_{1, \text { current }}\left(1+\frac{13}{2} \frac{t_{\text {age }}}{\tau_{\text {a,current }}^{\text {star }}},\right)^{2 / 13} \text {. }
$$

where $\tau_{\mathrm{a}, \text { current }}^{\text {star }}$ is given by Equation (24) at their current location.

In Figure 10 we show the initial period $P_{\text {initial }}$ for our sample of USP planets assuming $Q_{s} / k_{L, s}=10^{7}$ (circles) and the dashed lines indicate the range $Q_{s} / k_{L, s}=$ $\left[10^{6}, 10^{8}\right]$. We adopt $t_{\text {age }}=5 \mathrm{Gyr}$ and for the systems in which there is not a mass estimate (all but two systems), we use the mass-radius relation for cold, terrestrial-mass planets from Seager et al. 2007. The values of of $Q_{s} / k_{L, s}$ cover the wide range that has been inferred through ensemble analyses of star-planet systems (e.g., Hansen 2010, Penev et al. 2012 Collier Cameron \& Jardine 2018). In particular, the recent work by Penev et al. (2018) looking at the tidal spin up of Sun-like stars with hot Jupiters constrains $Q_{s} / k_{L, s} \sim 10^{7}$ for orbital periods of $\sim 1$ day.

We observe that only $3 / 44 \sim 7 \%(15 / 44 \sim 34 \%)$ of the systems could have migrated from $\gtrsim 1$ day to their current location inside 1 day for $Q_{s} / \mathbb{k}_{L, s}=10^{7}$ $\left(Q_{s} / k_{L, s}=10^{6}\right)$. Even with $Q_{s} / k_{L, s}=10^{6}$, the changes in periods are generally modest and the handful of planet with the most dramatic changes in periods tend to have higher effective temperatures, possibly indicating larger $Q_{s} / k_{L, s}$ since hotter stars have thinner convective envelopes (e.g., Ogilvie 2014).

We conclude that equilibrium tides on the star are unlikely to shrink the planetary orbits from initial periods of $\gtrsim 1$ days to their current locations and are, therefore, unable to account for the observed detachment from their outer companions. However, as pointed out by Lee \& Chiang (2017), tides on the star can do a good job at reproducing the steep fal 9 in frequency of USP planets with decreasing period observed by Sanchis-Ojeda et al. (2014). It is possible that the data are best explained by a combination of secular chaos delivering planets to $\sim 1$ day orbits and subsequent sculpting of the period distribution from tides raised on the star.

\subsubsection{Low-eccentricity migration in muti-planet systems}

Another possibility is that USP planets may have migrated inwards due to gravitational interactions with outer companions and gradual dissipation of orbital energy by tides acting on the planets (Mardling \& Lin 2004 Schlaufman et al. 2010). These systems follow loweccentricity paths during migration, different from our proposed model invoking high eccentricities.

\footnotetext{
9 In steady-state the equation 24 predicts that $d N / d P \propto$ $P^{10 / 3}$
}

For small eccentricities $\mathcal{F}(e) \simeq 7 e^{2} / 2$ in Equation 15 and $a_{\mathrm{f}} \simeq a$. Thus, Equation (14) becomes:

$$
\begin{aligned}
\tau_{a}^{\text {planet }} \simeq & 1.3 \times 10^{9} \text { yr }\left(\frac{Q_{p} / k_{L, p}}{100}\right)\left(\frac{1 R_{\oplus}}{R_{1}}\right)^{5}\left(\frac{M_{1}}{1 M_{\oplus}}\right) \\
& \times\left(\frac{P_{1}}{1 \text { day }}\right)^{13 / 3}\left(\frac{0.01}{e_{1}}\right)^{2} .
\end{aligned}
$$

This expression implies that the planet moves inwards as long as it can maintain an eccentricity at the percent level, which can be forced secularly ${ }^{10}$ by outer planets.

In this picture, the inner planet is subject to tidal dissipation, secular forcing from other planets, and apsidal precession from GR (and possibly other sources of precession). For a two-planet system Mardling \& Lin (2004) argued that the evolution behaves like that of a damped autonomous system that tends to align their apsidal orientations and reach a quasi-fixed point. At this state, the inner planet reaches an equilibrium eccentricity given by (Mardling 2007):

$$
e_{1}^{\mathrm{eq}}=\frac{5}{4} \frac{\left(a_{1} / a_{2}\right) e_{2}}{\left|1-\left(M_{1} / M_{2}\right)\left(a_{1} / a_{2}\right)^{1 / 2}+\gamma\right|},
$$

where

$$
\begin{aligned}
\gamma & \equiv \frac{4 G M_{s}}{c^{2} a} \frac{M_{s}}{M_{2}}\left(\frac{a_{2}}{a_{1}}\right)^{3} \frac{1}{\left(1-e_{1}^{2}\right)} \\
& \simeq 5.6\left(\frac{10 M_{\oplus}}{M_{2}}\right)\left(\frac{1 \text { day }}{P_{1}}\right)^{8 / 3}\left(\frac{P_{2}}{10 \text { day }}\right)^{2} .
\end{aligned}
$$

The fractional contribution that the relativistic potential makes to the apsidal advance, compared to the outer planet, is $\gamma /(1+\gamma)$. Thus, for a given perturber at $a_{2}$ with eccentricity $e_{2}$ we estimate the equilibrium eccentricity and replace this into $\tau_{a}^{\text {planet }}$ in Equation 26 to estimate the migration timescale.

For $\gamma \gg 1$, we can approximate $e^{\mathrm{eq}} \propto 1 / \gamma$, so we can plug $e^{\mathrm{eq}}$ into Equation 26 to obtain

$$
\begin{aligned}
& \tau_{a, \text { eq }}^{\text {planet }} \sim 6 \times 10^{9} \text { yr }\left(\frac{Q_{p} / k_{L, p}}{100}\right)\left(\frac{1 R_{\oplus}}{R_{1}}\right)^{5}\left(\frac{M_{1}}{1 M_{\oplus}}\right) \\
& \times\left(\frac{P_{1}}{1 \text { day }}\right)^{-7 / 3}\left(\frac{P_{2}}{10 \text { day }}\right)^{16 / 3}\left(\frac{10 M_{\oplus}}{M_{2}}\right)^{2}\left(\frac{0.1}{e_{2}}\right)^{2}(29)
\end{aligned}
$$

From this expression we conclude that this migration channel can work for $M_{2} \lesssim 10 M_{\oplus}$ only if $P_{2} \lesssim 10$ days. One example of a planetary system in this regime is CoRoT-7 which has two super-Earth planets inside 10 days (Léger et al. 2009). For Jupiter-mass planets the model can work for companions in much wider orbits $\left(P_{2} \lesssim 35\right.$ days $)$. Consistent with these estimates, Hansen \& Murray (2015) found that linear secular forcing in multi-planet systems is unable produce USP planets for $P_{2} \gtrsim 10$ days.

Based on the observed multiplicity in the Kepler sample, we argued in \$2 that most USP planets have Keplerlike companions beyond $\sim 20$ days. The possibility of

10 Mean-motion resonances can also force eccentricities against tidal dissipation, but the outer planets would have periods of less than a few days in this scenario, being unable to match the observations (Fig. 2]. 
having giants is disfavored by the lack of a metallicity trend for the USP planets' host stars (Winn et al. 2017). We conclude that low-eccentricity migration can account for some USP planets in relatively more compact configurations, but possibly not the majority.

\subsection{Predictions for TESS}

The soon-to-be launched Transiting Exoplanet Survey Satellite $(T E S S)$ by NASA is expected to find a couple dozen USP planets and hundreds of small planets $\left(\lesssim 2 R_{\oplus}\right)$ in short-period orbits $(\lesssim 10$ days $)$ around FGK stars (Sullivan et al. 2015). While a smaller sample size than that of Kepler, the TESS sample has the advantage that the host stars are much brighter and closer-in. This sample might allow testing some of our model predictions.

- For fast rotating host stars, the spin-orbit angle can potentially be measured using the RossiterMcLaughlin effect, as has been attempted for the USP $\rho 55$ Cancri e (Bourier \& Henrard 2014; López-Morales et al. 2014). Similarly, measurements of the orientation of the star's rotation axis can constrain the obliquity angles. Various techniques have been discussed, including ensemble analysis using projected rotational velocities (Schlaufman 2010, Morton \& Winn 2014) and individual systems using asteroseismology (Huber et al. 2013) or stellar spot crossing (Deming et al. 2011; Sanchis-Ojeda \& Winn 2011). A systematic misalignment (e.g., misalignment angle above $\sim 20^{\circ}$ ) would strongly support our model, especially if their longer-period counterparts have small misalignment angles.

- Follow-up radial velocity campaigns of USP planet systems may detect non-transiting companions in tens of days orbits, which are predicted by our model. We expect these companions to be typically more massive than the USP planets.

- Radial velocity follow-up of low-mass TESS planets (non-USP planets) may also determine their orbital eccentricities, a key ingredient in our model. We require that planets in low-multiple systems tend to have larger eccentricities, with the innermost planet having diffused to even higher eccentricities due to secular interactions $(e \sim 0.2-0.4)$. These planets should orbit beyond $P \sim 10$ days so tides do not generally damp their eccentricities $\left(\tau_{a}^{\text {planet }} \gg 10\right.$ Gyr in Eq. 26] $)$. We also expect to observe a population of eccentric planets in shortperiod orbits $(\lesssim 10$ days) that are excited by secular chaos with neighbouring planets and might have suffered some tidal decay as a consequence, but have yet to attain so high an eccentricity to experience tidal capture (\$5.1.2.

\section{SUMMARY}

If a large number of Kepler planetary systems contain a fair number of planets, possibly extending to $\gtrsim A U$ distances, and their orbits have non-negligible eccentricities and inclinations, we argue here that these systems can undergo large eccentricity and inclinations variations by non-linear secular interactions, through a process termed secular chaos. One of the consequences is the production of an ultra-short-period (USP) planet, as when the innermost planet (from periods of $5-10$ days) acquires high eccentricities from the interactions, it can approach the host star at such a close range that it is tidally captured onto a very short orbit (period short-ward of a day). We propose that most USP planets are formed by tidal high-eccentricity migration driven by secular chaos from periods of $\sim 5-10$ days.

Such a mechanism is intimately related to one of the mechanisms that has been proposed for forming hot Jupiters. In fact, the USP planets can be thought of as a scaled-down version of the hot Jupiter formation, both in planet masses, and in distance scales. For the latter, USP planets start their migration from around $\lesssim 0.1$ AU, while hot Jupiters from periods outward of $\sim \widetilde{1}$ AU. So while the latter need to reach extreme eccentricities $(e \gtrsim 0.98)$ to be tidally captured, a lower value $(e \gtrsim 0.8)$ is required for making USP planets.

Our scenario naturally explains the observation that most USP planets have distant companions with periods of $\sim 10-50$ days (Figs. 2 and 3), while their (not ultra)short-period counterparts $(\sim 1-3$ days $)$ reside in more compact configurations. We predict that USP planets orbit in inclined $\left(\sim 20^{\circ}-50^{\circ}\right)$ planes relative to those of both their outer companions and their host star's equator.

This scenario, even in cases where the eccentricity acquired by the innermost planet is not sufficiently high to be tidally captured, should, through gradual tidal circularization, bring the planets closer to the stars, perhaps forming some of the short-period planets (period of a couple days).

Future discoveries from TESS along with follow-up studies of systems with USP planets will reveal the prevalence of secular chaos in shaping the dynamical properties of USP planets.

Interestingly, the production of an USP planet could also happen in our very own Solar System in the future. The orbital evolution of Mercury is known to be chaotic with diffusion of the eccentricities on billion-year timescales. This diffusion can lead to Mercury colliding with Venus or the Sun within the next five billion years, before the Sun becomes a red giant (at 1\% probability, Laskar \& Gastineau 2009). Except in the latter case Mercury will not collide with the Sun - as its eccentricity gradually rises due to secular interactions, tidal capture will snare it away from the influences of other planets. A new ultra-short-period planet will be born and will become detectable by Kepler missions launched by alien civilizations.

\section{ACKNOWLEDGEMENTS}

We are grateful to Daniel Tamayo, Diego Muñoz, Eve Lee, Norm Murray, and Wei Zhu for enlightening discussions. C.P. acknowledges support from the Gruber Foundation Fellowship and Jeffrey L. Bishop Fellowship. Y. W. thanks NSERC for research support. This paper includes data collected by the Kepler mission. Funding for the Kepler mission is provided by the NASA Science Mission directorate. This research has made use of the NASA Exoplanet Archive, which is operated by the California Institute of Technology, under contract with the 
National Aeronautics and Space Administration under the Exoplanet Exploration Program.

\section{APPENDIX}

TRANSIT PROBABILITIES IN MULTI-PLANET SYSTEMS FROM TREMAINE \& DONG (2011)

We summarize the method by Tremaine \& Dong (2011) to estimate the transit probabilities in multi-planet systems. We only provide with the necessary details to reproduce our results in $\$ 2$ and invite the reader to consult Tremaine \& Dong (2011) for full explanations as we limit ourselves to providing the minimal amount of details required to reproduce our results.

As in Tremaine \& Dong (2011) we consider a system containing $n$ planets with semi-major axes specified by $\epsilon_{i}=$ $R_{s} / a_{i}$ with $i=1, . ., n$. The probability that a randomly oriented observer will detect $m$ transiting planets in this system is defined by $g_{\mathrm{mn}}\left(\epsilon_{1}, \ldots, \epsilon_{n}\right)$. For a single planet system we have the usual expression

$$
g_{11}(\epsilon)=\epsilon=\frac{R_{s}}{a} .
$$

For $n>1$ we assume that the inclinations $i$ relative to an arbitrary reference plane (e.g., the invariable plane) is given by a Fischer distribution

$$
q(i \mid \kappa)=\frac{\kappa \sin i}{2 \sinh \kappa} e^{\kappa \cos i}
$$

where the parameter $\kappa$ is related to the mean-square value of $\sin i$

$$
\left\langle\sin ^{2} i\right\rangle=\frac{2}{\kappa}\left(\operatorname{coth} \kappa-\frac{1}{\kappa}\right) .
$$

Then, the probability of a transit of a single planet, given the observer orientation $\cos \theta$ is

$$
u(x \mid \epsilon, \kappa)=\sum_{\ell=0}^{\infty} Q_{\ell}(\kappa) b_{\ell}(\epsilon) P_{\ell}(\cos \theta) .
$$

where $P_{\ell}$ is a Legendre polynomial,

$$
\begin{aligned}
Q_{\ell}(\kappa) & \equiv \int_{0}^{\pi} \operatorname{diq}(i \mid \kappa) P_{\ell}(\cos i), \quad Q_{0}=1, \\
& =\sqrt{\frac{\pi \kappa}{2}} \frac{I_{\ell+1 / 2}(\kappa)}{\sinh \kappa}
\end{aligned}
$$

with $I$ denoting a modified Bessel function and

$$
b_{\ell}(\epsilon)= \begin{cases}\epsilon, & \ell=0, \\ P_{\ell+1}(\epsilon)-P_{\ell-1}(\epsilon), & \ell \text { even, } \ell>0 \\ 0, & \ell \text { odd } .\end{cases}
$$

Two-planet system - Assuming that the intrinsic multiplicity is $n=2$, the probability that both transit for a random orientation of the observer is

$$
\begin{aligned}
g_{22}\left(\epsilon_{1}, \epsilon_{2}, \kappa\right) & =\frac{1}{2} \int_{-1}^{1} d \cos \theta u\left(\cos \theta \mid \epsilon_{1}, \kappa\right) u\left(\cos \theta \mid \epsilon_{2}, \kappa\right) \\
& =\sum_{\ell=0}^{\infty} \frac{Q_{\ell}^{2}(\kappa)}{2 \ell+1} b_{\ell}\left(\epsilon_{1}\right) b_{\ell}\left(\epsilon_{2}\right) .
\end{aligned}
$$

By denoting the inner planet as planet $1, \epsilon_{1}>\epsilon_{2}$, the probability that only the inner planet transits is

$$
\begin{aligned}
g_{12}^{\text {inner }}\left(\epsilon_{1}, \epsilon_{2}\right) & =\frac{1}{2} \int_{-1}^{1} d \cos \theta u\left(\cos \theta \mid \epsilon_{1}, \kappa\right)\left[1-u\left(\cos \theta \mid \epsilon_{2}, \kappa\right)\right] \\
& =g_{11}\left(\epsilon_{1}\right)-g_{22}\left(\epsilon_{1}, \epsilon_{2}, \kappa\right) .
\end{aligned}
$$

Thus, for an intrinsic two-planet system we can calculate the expected ratio of between the number of USP planets observed as single-transiting and those observed as multi-transiting systems as

$$
\left\langle\frac{f_{>1}}{f_{1}}\right\rangle=\frac{g_{22}\left(\epsilon_{1}, \epsilon_{2}, \kappa\right)}{\epsilon_{1}-g_{22}\left(\epsilon_{1}, \epsilon_{2}, \kappa\right)}
$$


Three or more planet systems - The expression in Equation (2) can be extended to higher intrinsic multiplicity systems using the formalism by Tremaine \& Dong (2011). Evidently, higher planet multiplicities will translate in higher values of $\left\langle f_{>1}^{\mathrm{USP}} / f_{1}\right\rangle$. We have not performed this analysis as it adds an extra degree of freedom and it requires knowledge of the intrinsic multiplicity of systems with USP planets.

\section{REFERENCES}

Adams E. R., Jackson B., Endl M., 2016, ApJ, 152, 47

Batalha, N. M., Borucki, W. J., Bryson, S. T., et al. 2011, ApJ, 729, 27

Bourier V., \& Henrard G., 2014, A\&A, 569, A65

Burke, C. J., Christiansen, J. L., Mullally, F., et al. 2015, ApJ, 809,8

Buchhave, L. A., Latham, D. W., Johansen, A., et al. 2012, Nature, 486, 375

Butler, R. P., Marcy, G. W., Williams, E., et al. 1997, ApJ, 474, 115

Collier Cameron, Andrew \& Jardine, Moira 2018, MNRAS, arXiv:1801.10561

Chiang, E., \& Laughlin, G. 2013, MNRAS, 431, 3444

D'Antona, F., \& Mazzitelli, I. 1994, ApJS, 90, 467

Dawson, R. I., \& Johnson, J. A. 2018, arXiv:1801.06117

Deming, D., P. V. Sada, B. Jackson, S. W.et al. 2011, ApJ, 740, 33

Dressing, C. D., \& Charbonneau, D. 2015, ApJ, 807, 45

Dumusque, X., Bonomo, A. S., Haywood, R. D., et al. 2014,

Fischer, D. A., \& Valenti, J. 2005, ApJ, 622, 1102

Fischer, D. A., Marcy, G. W., Butler, R. P., et al. 2008, ApJ, 675, 790

Foreman-Mackey D., Morton T. D., Hogg D. W., et al. 2016, AJ, 152,206

Fulton, B. J., Petigura, E. A., Howard, A. W., et al. 2017, AJ, 154,109

Gonzalez, G., 1997, MNRAS, 285, 403

Hamers, A. S., Antonini, F., Lithwick, Y., Perets, H. B., \& Portegies Zwart, S. F. 2017, MNRAS, 464, 688

Hansen, B. M. S. 2010, ApJ, 723, 285

Hansen, B. M. S., \& Murray, N. 2015, MNRAS, 448, 1044

Hansen, B. M. S., \& Zink, J. 2015, MNRAS, 450, 4505

Howard, A. W., Marcy, G. W., Bryson, S. T., et al. 2012, ApJS, 201,15

Huang, C., Wu, Y., \& Triaud, A. H. M. J. 2016, ApJ, 825, 98

Huber, D., Carter, J. A., Barbieri, M., et al. 2013, Sci, 342, 331

Hut P., 1981, A\&A, 99, 126

Jackson, B., Stark, C. C., Adams, E. R., et al. 2013, ApJ, 779, 165

Jackson, B., Jensen, E., Peacock, S., et al. 2016, CeMDA, 126, 227

Knutson, H. A., Fulton, B. J., Montet, B. T., et al. 2014, ApJ, 785,126

Lambeck, K., 1977, Philosophical Transactions of the Royal Society of London Series A, 287, 545

Laskar, J. 1996, CeMDA, 64, 115

Laskar, J. 1997, A\&A, 317, L75

Laskar, J., \& Gastineau, M. 2009, Nature, 459, 817

Lee, E. J., \& Chiang, E. 2017, ApJ, 842, 40

Léger, A., Rouan, D., Schneider, J., et al. 2009, A\&A, 506, 287

Lithwick, Y., \& Wu, Y. 2011, ApJ, 739, 31

Lithwick, Y., \& Wu, Y. 2014, PNAS, 111, 12610

Liu, B., Muñoz, D. J., \& Lai, D. 2015, MNRAS, 447, 747

López-Morales, M.; Triaud, A. H. M. J., Rodler, F. et al. 2014, ApJ, 792, L31

Lundkvist, M. S., Kjeldsen, H., Albrecht, S., et al. 2016, Nature Communications, 7, 11201

Mandell, A. M., Raymond, S. N., \& Sigurdsson, S. 2007, ApJ, $660,823 \mathrm{~b}$

Marcy, G. W., Butler, R. P., Fischer, D. A., et al. 2002, ApJ, 581, 1375

Marcy, G. W., Isaacson, H., Howard, A. W., Rowe, et al. 2014, ApJS, 210, 20
Mardling, R., \& Lin, D. N. C. 2002, ApJ, 573, 829

Mardling R. A., \& Lin D. N. C., 2004, ApJ, 614, 955

Mardling, R., 2007, MNRAS, 382, 1768

Mayor, M., Marmier, M., Lovis, C., et al. 2011, arXiv:1109.2497

McArthur, B. E., Endl, M., Cochran, W. D., et al. 2004, ApJ, 614,81

Morton, T. D., \& Winn, J. N. 2014, ApJ, 796, 47

Muñoz, D. J., Lai, D., \& Liu, B. 2016, MNRAS, 460, 1086

Murray-Clay, R. A., Chiang, E. I., \& Murray, N. 2009, ApJ, 693, 23

Nelson, B. E., Ford, E. B., Wright, J. T. Fischer, D. A., et al. MNRAS , 2014, 441, 442

Neron de Surgy, O.,\& Laskar, J., 1997, A\&A, 318, 975

Ogilvie, G. I. 2014, ARA\&A, 52, 171

Owen, J. E., \& Wu, Y. 2013, ApJ, 775, 105

Palla, F., \& Stahler, S. W. 1991, ApJ, 375, 288

Penev, K., Jackson, B., Spada, F., \& Thom, N. 2012, ApJ, 751, 96

Penev, K., Bouma, LG., Winn, J. N, \& Hartman, J. D. 2018, arXiv:1802.05269

Petigura, E. A., Marcy, G. W., Winn, J. N., et al. 2017b, ArXiv e-prints, arXiv: 1712.04042

Puranam, A. \& Batygin, K. 2018, arXiv:1802.08385

Queloz, D., Bouchy, F., Moutou, C., et al. 2009, A\&A, 506, 303

Rein, H., Liu, S-F., 2011, ApJ, 527

Rein, H., \& Tamayo, D. 2015, MNRAS, 452, 376

Rice, K. 2015, MNRAS, 448, 1729

Sanchis-Ojeda, R., Rappaport, S., Winn, J. N., et al. 2014, ApJ, 787,47

Sanchis-Ojeda, R. \& Winn, J. N., ApJ, 743, 61

Santos, N. C., Israelian, G., \& Mayor, M. 2004, A\&A, 415, 1153

Schlaufman, K. C., Lin, D. N. C. \& Ida, S. 2010, ApJ, 724, L53

Schlaufman, K. C. 2010, ApJ, 719, 602

Schlaufman, K. C., \& Laughlin, G. 2011, ApJ, 738, 177

Seager, S., Kuchner, M., Hier-Majumder, C.A., \& Militzer, B. 2007, ApJ, 669, 1279

Steffen, J. H., Ragozzine, D., Fabrycky, D. C., et al. 2012, PNAS, 109,7982

Steffen, J. H., \& Farr, W. M. 2013, ApJL, 774, L12

Steffen, J. H., \& Coughlin, J. L. 2016, PNAS, 113, 43

Steffen, J. H., \& Hwang, J. A. 2015, MNRAS, 448, 1956

Sterne, T. E. 1939, MNRAS, 99, 451

Sullivan, P. W., Winn, J. N., Berta-Thompson, Z. K. 2015, ApJ, 809, 77

Suzuki, D., Bennett, D.P., Sumi, T., et al. 2016, ApJ, 833, 145

Swift, J., Johnson, J. A., Morton, T. D., Crepp J. R., et al. 2013, ApJ, 764, 105

Tremaine, S., \& Dong, S. 2011, AJ, 143, 94

Udry, S., Mayor, M., Benz, W., et al. 2006, A\&A, 447, 361

Valencia, D., Ikoma, M., Guillot, T., et al. 2010, å, 516, A20

Valsecchi, F., Frederic, A. R., \& Steffen, J. H. 2014, ApJ, 793, 3

Vick, M., \& Lai, D. 2018, MNRAS,

Weiss, L. M., Rogers, L. A., Isaacson, H. T., et al. 2016, ApJ, 819,83

Winn, J. N., Sanchis-Ojeda, R., Rogers, L., et al. 2017, AJ, 154, 60

Wright, J. T., Marcy, G. W., Howard, A. W., et al. 2012, ApJ, 753,160

Wu, Y. 2018, AJ 155, 118

Wu, Y. \& Lithwick, Y. 2011, ApJ, 735,109

Xie, J.-W., Dong, S., Zhu, Z., et al. 2016, PNAS, 113, 11431

Zhu, W., Petrovich, C., Wu, Y. et al. 2018, arXiv:1802.09526 